Noname manuscript No.

(will be inserted by the editor)

\title{
Secant Update generalized version of PSB: a new approach
}

\author{
Nicolas Boutet - Rob Haelterman . \\ Joris Degroote
}

Received: date / Accepted: date

\begin{abstract}
In optimization, one of the main challenges of the widely used family of Quasi-Newton methods is to find an estimate of the Hessian matrix as close as possible to the real matrix. In this paper, we develop a new update formula for the estimate of the Hessian starting from the Powell-SymetricBroyden (PSB) formula and adding pieces of information from the previous steps of the optimization path. This lead to a multisecant version of PSB, which we call generalised PSB (gPSB), but which does not exist in general as was proven before. We provide a novel interpretation of this non-existence. In addition, we provide a formula that satisfies the multisecant condition and is as close to symmetric as possible and vice versa for a second formula. Subsequently, we add enforcement of the last secant equation and present a comparison between the different methods.
\end{abstract}

Keywords non-linear optimization $\cdot$ quasi-Newton formulae $\cdot$ multisecant equations $\cdot$ symmetric gradient

Mathematics Subject Classification (2010) 90C53, 49M15

\section{Introduction}

Today, solving complex problems has become a common situation in engineering. Numerical methods make it possible to solve problems that are increasingly complex and heavier, thanks to the combination of specific solver tools and the increasing available computational power. For most industrial

Nicolas Boutet · Rob Haelterman

Royal Military Academy, Dept. Mathematics, Renaissancelaan 30, 1000 Brussels, Belgium

Nicolas Boutet · Joris Degroote

Ghent University, Dept. of Electromechanical, Systems and Metal Engineering, SintPietersnieuwstraat 41, 9000 Ghent, Belgium

E-mail: nicolas.boutet@ugent.be 
or research and development applications, the computation can simply be considered as a black box for solving a problem based on given input variables.

However, when such a black box is available, a new question arises: which variables should be taken in order to optimize some objective function? Optimization is then a logical further step in those applications.

In this paper, we are solving problems expressed as the root finding problem

$$
\nabla g(\mathbf{x})=\mathbf{f}(\mathbf{x})=\mathbf{0}
$$

where $g: D_{F} \subset \mathbb{R}^{n} \rightarrow \mathbb{R}$ is the objective function of the optimization problem:

$$
\min _{\mathbf{x}} g(\mathbf{x})
$$

We assume the problem has the following characteristics:

(a) It is possible to calculate the objective function $g(\mathbf{x})$ with some code.

(b) Newton's method cannot be used because the analytic form of $g(\mathbf{x})$ is unknown, so the Hessian is not available.

(c) Methods like the adjoint state method [11, 16,32] make it possible to estimate the gradient of the problem $\nabla g(\mathbf{x})$.

(d) The problem, mainly the evaluation of $g(\mathbf{x})$ and $\nabla g(\mathbf{x})$, is computationally costly. This can be a consequence of the size of the problem (high dimensional) or of the complexity of the root finding problem even when it is of limited dimension. This leads to the choice of the required number of evaluations (or 'function calls') to reach convergence as a relevant measure of the performance of an algorithm.

\section{Quasi-Newton Least Change}

Based on the characteristic (b), as it is not possible to use the Jacobian of the gradient vector $\mathbf{f}(\mathbf{x})$, we use Quasi-Newton methods and try to estimate this matrix.

As the Jacobian matrix has to be estimated, one can define different properties that are suitable for this estimate. In most of the cases, imposing a selection of those properties is not sufficient to define one unique estimate for the Jacobian. The Least Change principle is then applied: the new estimate of the Jacobian is the matrix that has the desired properties and that is the closest to the previous estimate in a given norm. In the frame of our study, we use the Frobenius Norm for matrices and the Euclidean norm for vectors.

The choice of the estimate of the Jacobian matrix can thus be summarized as:

$$
\arg \min _{B_{i+1}} \frac{1}{2}\left\|B_{i}-B_{i+1}\right\|_{F}^{2}
$$

such that some properties (see below) are fulfilled

Quite a few papers in the literature give an overview of Quasi-Newton Least Change methods and on the properties that can be used in order to define a given method $[2,7,13,17,23,29,35]$. We center our attention on some 


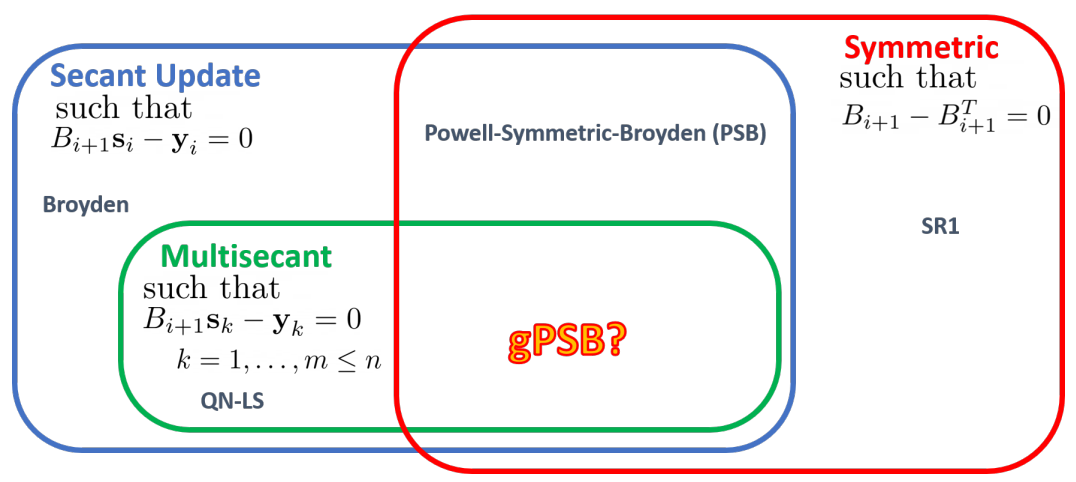

Fig. 1: Schematic view of some properties of Quasi-Newton Methods

of those potential properties listed below. Figure 1 gives a schematic overview of the listed properties.

- As we are working in optimization, the root finding problem is in fact the gradient of the objective function. The matrix $B_{i+1}$ is the Jacobian of a gradient, and therefore is a symmetric matrix (called Hessian matrix). This property can be written as $B_{i+1}=B_{i+1}^{T}$. One example of a Symmetric Least Change method is the Symmetric Rank One (SR1) [26].

- Another interesting property is called Secant Update. It consists in using the information available from the previous step on the optimization path by choosing the estimate such that $B_{i+1} \mathbf{s}_{i}=\mathbf{y}_{i}$ where $\mathbf{s}_{i}=\mathbf{x}_{i}-\mathbf{x}_{i-1}$ and $\mathbf{y}_{i}=\mathbf{f}\left(\mathbf{x}_{i}\right)-\mathbf{f}\left(\mathbf{x}_{i-1}\right)$. Broyden's good method is a well-known example of the application of this property $[5,6,12]$.

- The Secant Update property can be generalized to multiple previous points on the optimization path. The estimate is then made to satisfy multiple secant equations $B_{i+1} \mathbf{s}_{k}=\mathbf{y}_{k}$ for $k=1 \ldots m \leq n$. Grouping the vectors into a matrix, this can be reformulated as $B_{i+1} S_{i}=Y_{i}$. One can cite the Quasi-Newton Least Squares method as one example of a multisecant method [10, 20, 22, 25, 27,36].

One can try to combine some of those properties. For instance, the combination of the Least Change property, the symmetry and the Secant Update property leads to the Powell-Symmetric-Broyden (PSB) method $[33,34]$.

In order to maximize the quantity of information used when building the estimate of the Hessian matrix, our strategy is to improve PSB by combining the least change and multisecant properties with the symmetry. This should lead to a generalized PSB (gPSB) update formula that could be defined as:

$$
\begin{aligned}
\arg \min _{B_{i+1}} & \frac{1}{2}\left\|B_{i}-B_{i+1}\right\|_{F}^{2} \\
\text { such that } & B_{i+1}=B_{i+1}^{T} \\
& B_{i+1} S_{i}=Y_{i}
\end{aligned}
$$


The system (2.1) has already been investigated by Schnabel [37]. He proved however that the system can only be solved if $Y_{i}^{T} S_{i}$ is symmetric, which is a very restrictive condition. Indeed, it is true for quadratic function, but generally does not hold for other other problems. We can thus consider that in a global optimization context, it is impossible to build a gPSB update formula.

One strategy in order to tackle the impossibility of the construction of a generalized PSB update formula is to penalize the non-satisfaction of some of the properties using weight coefficients. This leads to methods like penalized PSB (pPSB) [19] or Secant Update penalized PSB (SUpPSB) [3]. In the former, all secant equations are imposed approximately while the latter imposes the latest secant condition exactly and the ones before approximately. However, those methods present a huge drawback: the definition of the weight coefficients has an important impact on the convergence of the method and tuning them is time-consuming for the user.

In Section 3, we approach the generalized PSB with alternating projections. This approach makes it possible to understand the impossibility stated in Theorem 3 in a different way. Moreover it provides two new gPSB update formulas: a multisecant version of PSB as close as possible of being symmetric and a symmetric version as close as possible of satisfying the multisecant condition.

Section 4 discusses an improvement of gPSB formulas of Section 3 by enforcing the Secant Update property, in the same way that SUpPSB improves pPSB by adding the Secant Update property [3].

\section{Alternating Projections applied on PSB}

\subsection{Alternating projections}

As it is in general not possible to build a generalized PSB update formula of the estimate of the Jacobian, we will approach this formula by imposing some properties and try to be as close as possible to satisfying some other ones. We start from PSB, which requires the following properties:

(1) Symmetric, $B_{i+1}=B_{i+1}^{T}$;

(2) Secant Update (satisfies the most recent secant equation), $B_{i+1} \mathbf{s}_{i}=\mathbf{y}_{i}$;

(3) Least Change (the new estimate of the Hessian is the closest to the previous one), $\left\|B_{i}-B_{i+1}\right\|_{F}^{2}$ is minimal.

We wish to develop an update formula that also satisfies the following additional property:

(4) The closest to satisfying multiple secant equations (closest to $B_{i+1} S_{i}=Y_{i}$ ).

We will use a method called the alternating projections to find the wanted formula. It is based on the successive projections $P_{i}$ of a point $x$ on different subspaces $\left(K_{i}\right)$. The projection is the map that associates the point $x$ to the point $P_{i} x \in K_{i}$, the closest to $x$. 
In our case, we will only use two subspaces, which will be chosen later. Then the alternating projection creates a sequence of matrices, alternatively one matrix belonging to $K_{1}$ and then one belonging to $K_{2}$. We note ${ }_{j} B$, the $j$-th projection of $B$ on one of the subspaces $K_{i}$. In the following section, we will add some extra subscripts to indicate the subspace onto which we project (e.g. ${ }_{j} B_{K_{1}}$ for the projection on $K_{1},{ }_{j} B_{M S}$ for the projection on multisecant matrices, ${ }_{j} \bar{B}$ for the projection on symmetric matrices...).

The sequence of projection is thus:

$$
{ }_{0} B \rightarrow{ }_{1} B_{K_{1}} \rightarrow{ }_{1} B_{K_{2}} \rightarrow{ }_{2} B_{K_{1}} \rightarrow{ }_{2} B_{K_{2}} \rightarrow{ }_{3} B_{K_{1}} \rightarrow{ }_{3} B_{K_{2}} \ldots
$$

In [4,8,31], it is proven that, if $K_{1}$ and $K_{2}$ are closed convex sets in a Hilbert space, the alternating projection converges to a fixed point that belongs to the intersection of $K_{1}$ and $K_{2}$. If $K_{1}$ and $K_{2}$ have no intersection, the alternating projection converges to a pair of fixed points (one belonging to $K_{1}$ and one belonging to $K_{2}$ ) projected on each other. The first point is then the point of $K_{1}$ the closest to $K_{2}$, and the second point belongs to $K_{2}$ and is the closest to $K_{1}$.

In our case, working with matrices, we have to project on closed convex subsets in $\mathbb{R}^{n \times n}$, which is a Euclidean space and thus a special case of a Hilbert space.

\subsection{Projections}

We will project on 2 sets:

- $K_{\text {Sym }}=\left\{A \in \mathbb{R}^{n \times n}: A=A^{T}\right\}:$ The set of symmetric matrices.

$-K_{M S}=\left\{A \in \mathbb{R}^{n \times n}: A S_{i}=Y_{i}\right\}$ : The set of matrices satisfying multiple given secant equations (we call them multisecant matrices).

We detail below the projections. The proofs that the associated sets are closed and convex are given in App B.1.

\subsubsection{Projection on the set of symmetric matrices}

In [28], it is shown that the projection onto the space of symmetric matrices is given by $P(A)=\frac{A+A^{T}}{2}$, if the norm is symmetric (which is the case for the Frobenius norm). This is the projection on $K_{S y m}$ :

$$
{ }_{j} \bar{B}=\frac{1}{2}\left({ }_{j} B+{ }_{j} B^{T}\right)
$$

\subsubsection{Projection on the set of multisecant matrices}

This projection must ensure the multiple secant equations. The formula for the projection on $K_{M S}$ is:

$$
{ }_{i+1} B={ }_{i} B+\left(Y_{i}-{ }_{i} B S_{i}\right)\left(S_{i}^{T} S_{i}\right)^{-1} S_{i}^{T}
$$


The proof of this formula is given in App B.2. The form of Formula (3.2) is very similar to Broyden's "good" method [5,6]. We can see it as a generalized version of Broyden's method.

\subsection{Generalized PSB}

Looking for the formula of generalized PSB, the problem can be expressed as: Let $B_{i} \in \mathbb{R}^{n \times n}, Y_{i}$ and $S_{i} \in \mathbb{R}^{n \times m}$, with $m \leq n$ and $S_{i}$ full-rank. We search for $B_{i+1}$ such that:

1. $B_{i+1} S_{i}=Y_{i}$

2. $B_{i+1}=B_{i+1}^{T}$

3. $\left\|B_{i}-B_{i+1}\right\|_{F}^{2}$ is minimal

Condition 1 above corresponds to the set $K_{M S}$ and condition 2 to the set $K_{\text {Sym }}$. As there is a formula fulfilling the condition 1 (QN-LS) [22, 23] and one fulfilling the condition 2 (SR1) [26], but none fulfilling both together in general [37], we can deduce that the set $K_{S y m}$ and $K_{M S}$ have no intersection. Applying the alternating projection with those two sets then leads to a pair of matrices projected on each other. Those matrices will be used to define the update formula for the Hessian. We call those formulas:

- gPSB Sym (generalized PSB Symmetric, Theorem 1)

- gPSB MS (generalized PSB MultiSecant, Theorem 2).

The form of the formulas are given in the two following theorems. We handle both theorems in a single proof in Appendix B.3. We note that gPSB Sym (Theorem 1) is the formula given by Schnabel [37].

In the following theorems, we use the Moore-Penrose pseudoinverse $S^{+} S=$ $I$ where $S^{+}=\left(S^{T} S\right)^{-1} S^{T}$. Those formulas require the computation of the inverse of a matrix. Based on characteristic (d) in Section 1, we consider that the cost of this inversion is negligible compared to the calculation of the function value and gradient value. The complexity is $\mathcal{O}\left(n^{3}\right)$ and can be reduced to $\mathcal{O}(n)$ with some proper parallel algorithm [9].

Theorem 1 (gPSB Sym) Let $B_{i} \in \mathbb{R}^{n \times n}, Y_{i}$ and $S_{i} \in \mathbb{R}^{n \times m}$, with $m \leq n$ and $S_{i}$ full-rank. Let $K_{S y m \triangleright M S}$ be the set of matrices $B$ such that:

- $B$ is symmetric

- $B$ is the closest to the set $K_{M S}=\left\{A \in \mathbb{R}^{n \times n}: A S_{i}=Y_{i}\right\}$

Then, $B_{i+1} \in K_{\text {Sym } \triangleright M S}$ such that $\left\|B_{i+1}-B_{i}\right\|_{F}$ is minimal is given by:

$$
\begin{aligned}
B_{i+1}= & \frac{B_{i}^{T}}{2}+\frac{B_{i}}{2}-\frac{B_{i}^{T} S_{i} S_{i}^{+}}{2}-\frac{\left(S_{i}^{+}\right)^{T} S_{i}^{T} B_{i}}{2}-\frac{B_{i} S_{i} S_{i}^{+}}{2} \\
& -\frac{\left(S_{i}^{+}\right)^{T} S_{i}^{T} B_{i}^{T}}{2}+\frac{\left(S_{i}^{+}\right)^{T} S_{i}^{T} B_{i}^{T} S_{i} S_{i}^{+}}{2}+\frac{\left(S_{i}^{+}\right)^{T} S_{i}^{T} B_{i} S_{i} S_{i}^{+}}{2} \\
& +Y_{i} S_{i}^{+}+\left(S_{i}^{+}\right)^{T} Y_{i}^{T}-\frac{\left(S_{i}^{+}\right)^{T} S_{i}^{T} Y_{i} S_{i}^{+}}{2}-\frac{\left(S_{i}^{+}\right)^{T} Y_{i}^{T} S_{i} S_{i}^{+}}{2}
\end{aligned}
$$


Theorem 2 (gPSB MS) Let $B_{i} \in \mathbb{R}^{n \times n}, Y_{i}$ and $S_{i} \in \mathbb{R}^{n \times m}$, with $m \leq n$ and $S_{i}$ full-rank. Let $K_{M S \triangleright S y m}$ be the set of matrices $B$ such that:

$-B S_{i}=Y_{i}$

- $B$ is the closest to the set $K_{S y m}=\left\{A \in \mathbb{R}^{n \times n}: A=A^{T}\right\}$

Then, $B_{i+1} \in K_{M S \triangleright S y m}$ such that $\left\|B_{i+1}-B_{i}\right\|_{F}$ is minimal is given by:

$$
\begin{aligned}
B_{i+1}= & \frac{B_{i}^{T}}{2}+\frac{B_{i}}{2}-\frac{B_{i}^{T} S_{i} S_{i}^{+}}{2}-\frac{\left(S_{i}^{+}\right)^{T} S_{i}^{T} B_{i}}{2}-\frac{B_{i} S_{i} S_{i}^{+}}{2} \\
& -\frac{\left(S_{i}^{+}\right)^{T} S_{i}^{T} B_{i}^{T}}{2}+\frac{\left(S_{i}^{+}\right)^{T} S_{i}^{T} B_{i}^{T} S_{i} S_{i}^{+}}{2}+\frac{\left(S_{i}^{+}\right)^{T} S_{i}^{T} B_{i} S_{i} S_{i}^{+}}{2} \\
& +Y_{i} S_{i}^{+}+\left(S_{i}^{+}\right)^{T} Y_{i}^{T}-\left(S_{i}^{+}\right)^{T} Y_{i}^{T} S_{i} S_{i}^{+}
\end{aligned}
$$

\subsection{Existence conditions of gPSB}

The two formulas of Theorem 1 and 2 are different. This confirms that the subspaces $K_{S y m}$ and $K_{M S}$ do not have any point in common except if $Y_{i}^{T} S_{i}$ is symmetric.

The necessary and sufficient condition for existence of a gPSB formula given by Schnabel [37], which can be written as Theorem 3, can be easily checked based on the two formulas given in the theorems of the previous section.

Theorem 3 Let $Y_{i}$ and $S_{i} \in \mathbb{R}^{n \times m}$, with $m \leq n$ and $S_{i}$ full-rank. There exists $B_{i+1} \in \mathbb{R}^{n \times n}$ such that:

$-B_{i+1} S_{i}=Y_{i}$

$-B_{i+1}=B_{i+1}^{T}$

- $\left\|B_{i}-B_{i+1}\right\|_{F}^{2}$ is minimal

if and only if $Y_{i}^{T} S_{i}$ is symmetric.

This result seems however to be in contradiction with a simple reasoning about the degrees of freedom. Indeed, when $m$ is small enough (as is often the case) the system is under-determined - so the $B$ we get lies in some subspace of dimension $>1$ assuming the equations are consistent. Then the minimization (least change condition) should give an unique solution from this subspace. The condition that $Y_{i}^{T} S_{i}$ need to be symmetric, is however very restrictive, as it generally only happens for quadratic functions. So proving that this solution only exists under quite strict conditions seems to be paradoxical.

In Appendix B.4.1, we give an alternative proof of Theorem 3, which makes it easier to understand this apparent paradox by proving that the equations are not consistent. The proof shows that there is at least one linear dependency for the system $B S=Y$ and $B=B^{T}$ when $m=2$. Note that this result is not dependent on the Least Change condition. This leads to Theorem 4, which is an extension of Theorem 3, and counts the number of linear dependencies for higher values of $m$. Its proof is given in Appendix B.4.2. 
Table 1: Overview of the PSB formulas

\begin{tabular}{lcccc}
\hline Formula & Secant Update & Multisecant & Symmetric & Reference \\
\hline \hline PSB & YES & & YES & {$[33,34]$} \\
\hline gPSB & YES & YES & YES & Not possible [37] \\
\hline gPSB Sym & & $\begin{array}{c}\text { As close } \\
\text { as possible }\end{array}$ & YES & Theorem 1 \\
\hline gPSB MS & YES & $\begin{array}{c}\text { As close } \\
\text { as possible }\end{array}$ & Theorem 2 \\
\hline SUgPSB Sym & YES & $\begin{array}{c}\text { As close } \\
\text { as possible }\end{array}$ & YES & Theorem 5 \\
\hline SUgPSB MS & YES & YES & As close as possible & Theorem 6 \\
\hline \hline
\end{tabular}

Theorem 4 Let $Y_{m}$ and $S_{m} \in \mathbb{R}^{n \times m}$, with $m \leq n$ and $S_{i}$ full-rank. We consider the system of $n\left(m+\frac{n-1}{2}\right)$ equations with $\overline{n^{2}}$ unknowns resulting from the problem of finding $B \in \mathbb{R}^{n \times n}$ such that:

$$
\left\{\begin{aligned}
B S_{m} & =Y_{m} \\
B & =B^{T}
\end{aligned}\right.
$$

This system has $\frac{m(m-1)}{2}$ equations that can be expressed as a linear combination of the other equations. So for $m>1$, the linear system is not consistent.

\section{Secant Update generalized PSB}

If we want to maximize the amount of information from the previous steps when building the estimate of the Hessian matrix, we can improve the two formulas from the previous Section. Indeed, in the same way that PSB can combine the symmetry and the secant update property, the gPSB Sym could be improved such that the last secant equation would be fulfilled. Therefore, we will apply the alternating projection but instead of projecting on the set $K_{S y m}$, we will project on the set $K_{S y m S U}$ which, as PSB, combines the symmetry and the secant update.

To gPSB Sym (Theorem 1), we add the requirement that the last secant equation needs to be satisfied exactly. This leads to Theorem 5 (SUgPSB Sym). To gPSB MS (Theorem 2), we add the requirement that the approximate Hessian needs to be the closest possible to the set of symmetric matrices satisfying the last secant equation, instead of simply being the closest to the set of symmetric matrices. This will lead to Theorem 6 (SUgPSB MS). A summary of those formulas and the corresponding properties is given in Table 1 .

To do this, we will alternatively project on the set of symmetrical matrices satisfying the last secant equation and on the set of matrices satisfying multiple 
secant equations. We also show that under certain conditions SUgPSB Sym and SUgPSB MS will be identical.

\subsection{Projections}

We first need to define three sets:

- $K_{S U}$ : for the set of secant update matrices. This is a special case of $K_{M S}$ where one only considers one secant equation.

- $K_{S y m S U}$ : The set of symmetric Secant Update matrices $\left(K_{S y m} \cap K_{S U}\right)$, which is in fact the formula of PSB with non-symmetrical start, written ${ }_{j} B_{S y m S U}$.

- $K_{M S \triangleright S y m}$ : We use the Theorem 2 (gPSB MS) for the projection on the set of multisecant matrices, written ${ }_{j} B_{M S \triangleright S y m}$. This differs from the previous section $\left(K_{M S}\right)$ as the new matrix ${ }_{j} B_{M S \triangleright S y m}$ has one additional property: being the closest to the set $K_{\text {Sym }}=\left\{A \in \mathbb{R}^{n \times n}: A=A^{T}\right\}$. It is in fact the projection onto:

$$
K_{M S \triangleright S y m}=\left\{A \in \mathbb{R}^{n \times n}: A S_{i}=Y_{i} \text { and } \mathrm{A} \text { is the closest to } K_{S y m}\right\}
$$

We detail below the projections. The proofs that the associated sets are closed and convex ae given in App D.1.

\subsubsection{Projection on the set of symmetric secant update matrices}

This projection is in fact a PSB. However the standard formula of PSB starts from a symmetric matrix. We need a form from a non-symmetric matrix. The projection is given by:

$$
{ }_{i+1} B={ }_{i} \bar{B}+\frac{\overline{\mathbf{w}}_{i} \mathbf{s}_{i}^{T}}{\mathbf{s}_{i}^{T} \mathbf{s}_{i}}+\frac{\mathbf{s}_{i} \overline{\mathbf{w}}_{i}^{T}}{\mathbf{s}_{i}^{T} \mathbf{s}_{i}}-\frac{\overline{\mathbf{w}}_{i}^{T} \mathbf{s}_{i}}{\left(\mathbf{s}_{i}^{T} \mathbf{s}_{i}\right)^{2}} \mathbf{s}_{i} \mathbf{s}_{i}^{T}
$$

where $\overline{\mathbf{w}}_{i}=\mathbf{y}_{i}-{ }_{i} \bar{B} \mathbf{s}_{i}$. The proof of this formula is given in App D.2.

\subsubsection{Projection on the set of multisecant matrices}

For the multisecant projection, we can reuse the formula of Theorem 2 (gPSB MS). Indeed, this formula already contains the property "closest to a symmetric matrix". We can see this last formula as the projection onto:

$$
K_{M S \triangleright S y m}=\left\{A \in \mathbb{R}^{n \times n}: A S_{i}=Y_{i} \text { and } \mathrm{A} \text { is the closest to } K_{S y m}\right\}
$$




\subsection{SUgPSB}

In the same way as we have defined a pair of formulas for gPSB, we can now define a pair of formulas of SUgPSB.

On the one side, we have a symmetric version of SUgPSB being the closest to the set of multisecant matrices: Theorem 5 . On the other side, we have the SUgPSB being multisecant and the closest to the set of the symmetric SU matrices: Theorem 6 . Note that having the multisecant property implies the latter also have the secant update property. The proof of both theorems is given in Apprendix D.3.

Theorem 5 (SUgPSB Sym) Let $B_{i} \in \mathbb{R}^{n \times n}, Y_{i}$ and $S_{i} \in \mathbb{R}^{n \times m}$ with $m \leq n, \mathbf{s}_{i}$ the last column of $S_{i}, \mathbf{y}_{i}$ the last column of $Y_{i}$ and $S_{i}$ full-rank. Let $K_{\text {SymSUcMS }}$ be the set of matrices $B$ such that:

$-B$ is symmetric

$-B \mathbf{s}_{i}=\mathbf{y}_{i}$

- $B$ is the closest to the set $K_{M S}=\left\{A \in \mathbb{R}^{n \times n}: A S_{i}=Y_{i}\right\}$

Then, $B_{i+1} \in K_{\text {SymSUcMS }}$, such that $\left\|B_{i+1}-B_{i}\right\|_{F}$ is minimal, is given by:

$$
\begin{aligned}
B_{i+1}= & \frac{B_{i}^{T}}{2}+\frac{B_{i}}{2}-\frac{B_{i}^{T} S_{i} S_{i}^{+}}{2}-\frac{\left(S_{i}^{+}\right)^{T} S_{i}^{T} B_{i}}{2}-\frac{B_{i} S_{i} S_{i}^{+}}{2} \\
& -\frac{\left(S_{i}^{+}\right)^{T} S_{i}^{T} B_{i}^{T}}{2}+\frac{\left(S_{i}^{+}\right)^{T} S_{i}^{T} B_{i}^{T} S_{i} S_{i}^{+}}{2}+\frac{\left(S_{i}^{+}\right)^{T} S_{i}^{T} B_{i} S_{i} S_{i}^{+}}{2} \\
& +Y_{i} S_{i}^{+}+\left(S_{i}^{+}\right)^{T} Y_{i}^{T}-\frac{\left(S_{i}^{+}\right)^{T} S_{i}^{T} Y_{i} S_{i}^{+}}{2}-\frac{\left(S_{i}^{+}\right)^{T} Y_{i}^{T} S_{i} S_{i}^{+}}{2} \\
& +\frac{\left(S_{i}^{+}\right)^{T} S_{i}^{T} \mathbf{y}_{i} \mathbf{s}_{i}^{T}}{2 \mathbf{s}_{i}^{T} \mathbf{s}_{i}}+\frac{\mathbf{s}_{i} \mathbf{y}_{i}^{T} S_{i} S_{i}^{+}}{2 \mathbf{s}_{i}^{T} \mathbf{s}_{i}}-\frac{\left(S_{i}^{+}\right)^{T} Y_{i}^{T} \mathbf{s}_{i} \mathbf{s}_{i}^{T}}{2 \mathbf{s}_{i}^{T} \mathbf{s}_{i}}-\frac{\mathbf{s}_{i} \mathbf{s}_{i}^{T} Y_{i} S_{i}^{+}}{2 \mathbf{s}_{i}^{T} \mathbf{s}_{i}}
\end{aligned}
$$

Theorem 6 (SUgPSB MS) Let $B_{i} \in \mathbb{R}^{n \times n}, Y_{i}$ and $S_{i} \in \mathbb{R}^{n \times m}$ with $m \leq n, \mathbf{s}_{i}$ the last column of $S_{i}, \mathbf{y}_{i}$ the last column of $Y_{i}$ and $S_{i}$ full-rank. Let $K_{M S \triangleright S y m S U}$ be the set of matrices $B$ such that:

$-B_{i+1} S_{i}=Y_{i}$

- $B_{i+1}$ is the closest to the set $\left(K_{S U} \cap K_{S y m}\right)=\left\{A \in \mathbb{R}^{n \times n}: A \mathbf{s}_{i}=\mathbf{y}_{i}\right.$ and $\left.A=A^{T}\right\}$

Then, $B_{i+1} \in K_{M S \triangleright S y m S U}$, such that $\left\|B_{i+1}-B_{i}\right\|_{F}$ is minimal, is given by:

$$
\begin{aligned}
B_{i+1}= & \frac{B_{i}^{T}}{2}+\frac{B_{i}}{2}-\frac{B_{i}^{T} S_{i} S_{i}^{+}}{2}-\frac{\left(S_{i}^{+}\right)^{T} S_{i}^{T} B_{i}}{2}-\frac{B_{i} S_{i} S_{i}^{+}}{2} \\
& -\frac{\left(S_{i}^{+}\right)^{T} S_{i}^{T} B_{i}^{T}}{2}+\frac{\left(S_{i}^{+}\right)^{T} S_{i}^{T} B_{i}^{T} S_{i} S_{i}^{+}}{2}+\frac{\left(S_{i}^{+}\right)^{T} S_{i}^{T} B_{i} S_{i} S_{i}^{+}}{2} \\
& +Y_{i} S_{i}^{+}+\left(S_{i}^{+}\right)^{T} Y_{i}^{T}-\left(S_{i}^{+}\right)^{T} Y_{i}^{T} S_{i} S_{i}^{+}
\end{aligned}
$$


4.3 Equivalence condition

As summarized in Table 1, we have developed 2 pairs of formulas:

1. Two version of gPSB, being the closest to each other:

- gPSB Sym $\in K_{S y m}=\left\{A \in \mathbb{R}^{n \times n}: A=A^{T}\right\}$, Theorem 1

- gPSB MS $\in K_{M S}=\left\{A \in \mathbb{R}^{n \times n}: A S_{i}=Y_{i}\right\}$, Theorem 2

2. Two version of SUgPSB, being the closest to each other:

$-\mathrm{SUgPSB}$ Sym $\in K_{S y m S U}=\left\{A \in \mathbb{R}^{n \times n}: A \mathbf{y}_{i}=\mathbf{s}_{i}\right.$ and $\left.A=A^{T}\right\}$,

Theorem 5

- SUgPSB MS $\in K_{M S \triangleright S y m S U}=\left\{A \in \mathbb{R}^{n \times n}: A S_{i}=Y_{i}\right.$ and A is the closest to $\left.K_{S U} \cap K_{S y m}\right\}$, Theorem 6

We will now compare them. Figure 2 gives a overview of the different update formulas and the equivalence conditions.

\subsubsection{Comparison SUgPSB Sym and SUgPSB MS}

We can now verify that formulas of Theorem 5 (SUgPSB Sym) and 6 (SUgPSB MS) are equal if and only if $S_{i} Y_{i}^{T}=Y_{i} S_{i}^{T}$. This is in fact another alternative proof for Theorem 3 .

Theorem 7 The formulas of Theorem 5 (SUgPSB Sym) and 6 (SUgPSB MS) are equal if and only if $S_{i}^{T} Y_{i}$ is equal to $Y_{i}^{T} S_{i}$.

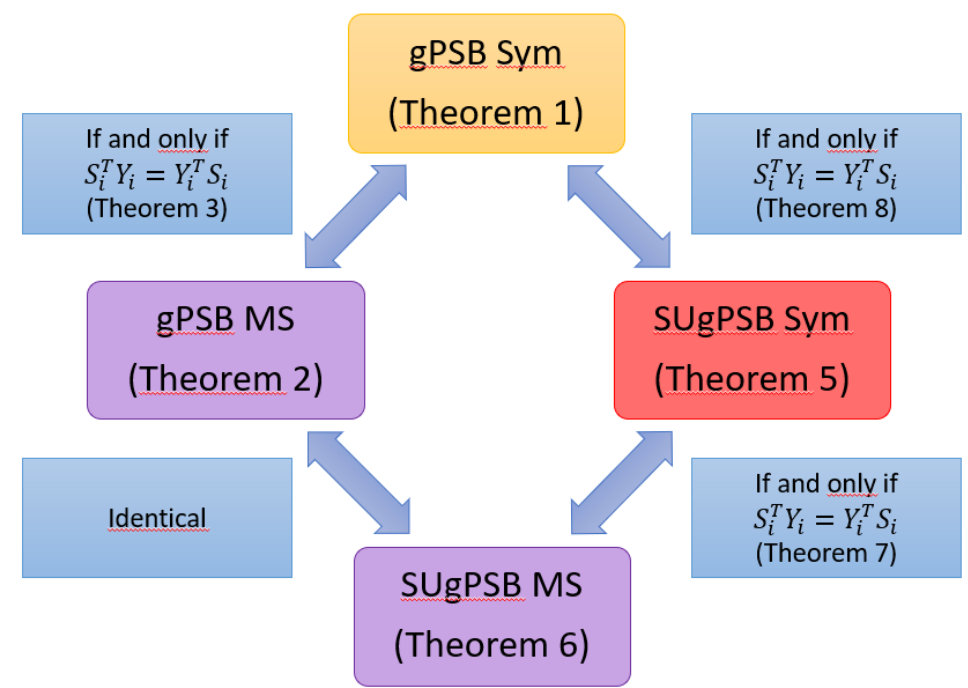

Fig. 2: Schematic view of equivalence conditions between the formulas 
Proof $\Leftarrow$ If $B_{i+1}$ is symmetric and multisecant, formulas of Theorem 5 (SUgPSB Sym) and 6 (SUgPSB MS) must be equal. Keeping only the differences between the formulas, we have:

$$
\begin{aligned}
& \frac{\left(S_{i}^{+}\right)^{T} S_{i}^{T} \mathbf{y}_{i} \mathbf{s}_{i}^{T}}{2 \mathbf{s}_{i}^{T} \mathbf{s}_{i}}-\frac{\left(S_{i}^{+}\right)^{T} S_{i}^{T} Y_{i} S_{i}^{+}}{2}-\frac{\left(S_{i}^{+}\right)^{T} Y_{i}^{T} \mathbf{s}_{i} \mathbf{s}_{i}^{T}}{2 \mathbf{s}_{i}^{T} \mathbf{s}_{i}} \\
& -\frac{\left(S_{i}^{+}\right)^{T} Y_{i}^{T} S_{i} S_{i}^{+}}{2}-\frac{\mathbf{s}_{i} \mathbf{s}_{i}^{T} Y_{i} S_{i}^{+}}{2 \mathbf{s}_{i}^{T} \mathbf{s}_{i}}+\frac{\mathbf{s}_{i} \mathbf{y}_{i}^{T} S_{i} S_{i}^{+}}{2 \mathbf{s}_{i}^{T} \mathbf{s}_{i}}=-\left(S_{i}^{+}\right)^{T} Y_{i}^{T} S_{i} S_{i}^{+}
\end{aligned}
$$

As the equation must be true for every value of $\mathbf{s}_{i}$, we must have:

$$
\left\{\begin{array}{l}
\frac{\left(S_{i}^{+}\right)^{T} S_{i}^{T} Y_{i} S_{i}^{+}}{2}-\frac{\left(S_{i}^{+}\right)^{T} Y_{i}^{T} S_{i} S_{i}^{+}}{2}=-\left(S_{i}^{+}\right)^{T} Y_{i}^{T} S_{i} S_{i}^{+} \\
\left(S_{i}^{+}\right)^{T} S_{i}^{T} \mathbf{y}_{i} \mathbf{s}_{i}^{T}-\left(S_{i}^{+}\right)^{T} Y_{i}^{T} \mathbf{s}_{i} \mathbf{s}_{i}^{T}-\mathbf{s}_{i} \mathbf{s}_{i}^{T} Y_{i} S_{i}^{+}+\mathbf{s}_{i} \mathbf{y}_{i}^{T} S_{i} S_{i}^{+}=0
\end{array}\right.
$$

The first condition leads to $Y_{i}^{T} S_{i}=S_{i}^{T} Y_{i}$ which is enough to fulfill the second condition.

$\Rightarrow$ If $Y_{i}^{T} S_{i}=S_{i}^{T} Y_{i}$, we also have $\mathbf{y}_{i}^{T} S_{i}=\mathbf{s}_{i}^{T} Y_{i}$ (the last line of $Y_{i}^{T} S_{i}$ ) and $S_{i}^{T} \mathbf{y}_{i}=Y_{i}^{T} \mathbf{s}_{i}$ (its last column). Replacing this in Theorem 5 (SUgPSB Sym) gives Theorem 6 (SUgPSB MS).

\subsubsection{Comparison gPSB and SUgPSB}

First, we can note that the formulas of Theorems 2 (gPSB MS) and Theorem 6 (SUgPSB MS) are identical. This is logical as the multisecant formula the closest to the SU symmetric is already a secant update. So forcing the fact that the formula should be the closest to a secant update has no impact.

In Theorem 3, we've already seen that gPSB exists if and only if $Y_{i}^{T} S_{i}$ is symmetric. Indeed, then formulas of Theorem 1 (gPSB Sym) and Theorem 2 (gPSB MS) are equal. In Theorem 7, we have also seen the same for SUgPSB using Theorem 5 (SUgPSB Sym) and Theorem 6 (gPSB Sym). We now look for equality conditions for the symmetric update formulas.

Theorem 8 The formulas of Theorems 1 (gPSB Sym) and 5 (SUgPSB Sym) are equal if and only if the last column of $S_{i}^{T} Y_{i}$ is equal to the last column of $Y_{i}^{T} S_{i}$.

Proof $\Rightarrow$ If both formulas are equal, we compare Theorem 1 (gPSB Sym) and Theorem 5 (SUgPSB Sym). After simplification of identical terms, we have:

$$
\begin{aligned}
0 & =\frac{\left(S_{i}^{+}\right)^{T} S_{i}^{T} \mathbf{y}_{i} \mathbf{s}_{i}^{T}}{2 \mathbf{s}_{i}^{T} \mathbf{s}_{i}}-\frac{\left(S_{i}^{+}\right)^{T} Y_{i}^{T} \mathbf{s}_{i} \mathbf{s}_{i}^{T}}{2 \mathbf{s}_{i}^{T} \mathbf{s}_{i}}-\frac{\mathbf{s}_{i} \mathbf{s}_{i}^{T} Y_{i} S_{i}^{+}}{2 \mathbf{s}_{i}^{T} \mathbf{s}_{i}}+\frac{\mathbf{s}_{i} \mathbf{y}_{i}^{T} S_{i} S_{i}^{+}}{2 \mathbf{s}_{i}^{T} \mathbf{s}_{i}} \\
& =\left(S_{i}^{+}\right)^{T} S_{i}^{T} \mathbf{y}_{i} \mathbf{s}_{i}^{T}-\left(S_{i}^{+}\right)^{T} Y_{i}^{T} \mathbf{s}_{i} \mathbf{s}_{i}^{T}-\mathbf{s}_{i} \mathbf{s}_{i}^{T} Y_{i} S_{i}^{+}+\mathbf{s}_{i} \mathbf{y}_{i}^{T} S_{i} S_{i}^{+} \\
& =S_{i}^{T} \mathbf{y}_{i} \mathbf{s}_{i}^{T} S_{i}-Y_{i}^{T} \mathbf{s}_{i} \mathbf{s}_{i}^{T} S_{i}-S_{i}^{T} \mathbf{s}_{i} \mathbf{s}_{i}^{T} Y_{i}+S_{i}^{T} \mathbf{s}_{i} \mathbf{y}_{i}^{T} S_{i} \\
& =\left(S_{i}^{T} \mathbf{y}_{i}-Y_{i}^{T} \mathbf{s}_{i}\right) \mathbf{s}_{i}^{T} S_{i}+S_{i}^{T} \mathbf{s}_{i}\left(\mathbf{y}_{i}^{T} S_{i}-\mathbf{s}_{i}^{T} Y_{i}\right) \\
& =\left(S_{i}^{T} \mathbf{y}_{i}-Y_{i}^{T} \mathbf{s}_{i}\right) \mathbf{s}_{i}^{T} S_{i}+\left(\left(S_{i}^{T} \mathbf{y}_{i}-Y_{i}^{T} \mathbf{s}_{i}\right) \mathbf{s}_{i}^{T} S_{i}\right)^{T}
\end{aligned}
$$


$\left(S_{i}^{T} \mathbf{y}_{i}-Y_{i}^{T} \mathbf{s}_{i}\right) \mathbf{s}_{i}^{T} S_{i}$ is skew-symmetric, so its rank must be even. But $\left(S_{i}^{T} \mathbf{y}_{i}-\right.$ $\left.Y_{i}^{T} \mathbf{s}_{i}\right) \mathbf{s}_{i}^{T} S_{i}$ has the form of $\mathbf{u v}^{T}$. Its rank is at most 1 .

We deduce that its rank must be 0 which is only possible if $\mathbf{u}=\mathbf{0}$ or $\mathbf{v}=\mathbf{0}$. $\mathbf{s}_{i}^{T} S_{i}$ cannot be null because by hypothesis $S_{i}$ is full-rank. So we have:

$$
S_{i}^{T} \mathbf{y}_{i}-Y_{i}^{T} \mathbf{s}_{i}=\mathbf{0}
$$

This is equivalent to say that the last column of $S_{i}^{T} Y_{i}$ is equal to the last column of $Y_{i}^{T} S_{i}$.

$\Leftarrow$ If the last column of $S_{i}^{T} Y_{i}$ is equal to the last column of $Y_{i}^{T} S_{i}$, we have $S_{i}^{T} \mathbf{y}_{i}=Y_{i}^{T} \mathbf{s}_{i}$ and $\mathbf{y}_{i}^{T} S_{i}=\mathbf{s}_{i}^{T} Y_{i}$. The equality is then quickly verified.

The equality condition of the last column of $S_{i}^{T} Y_{i}$ is obviously fulfilled when $Y_{i}^{T} S_{i}=S_{i}^{T} Y_{i}$. Then formulas of Theorems 2 (gPSB MS), 1 (gPSB Sym) and 5 (SUgPSB Sym) are identical. This is then our gPSB formula.

\section{Numerical experiments}

This section is devoted to numerical experiments. In the following subsection we give an overview of our problem set and the algorithm implementation. We will mainly follow the sections of the best practices for comparing optimization algorithms [1] to describe our experimental set. Then in the second subsection, we present and discuss our results. Finally, we give a short overview of potential future improvement.

\subsection{Problem set and implementation}

\subsubsection{Test set}

The robustness of the algorithms was tested on a set of 146 unconstrained problems of the type "sum of squares" from the CUTEst collection [18]. For problems where the dimension is a parameter, we took the highest dimension from the standard values given in CUTEst where at least one of the tested algorithms could solve the problem.

Next to the standard starting points provided with the test problems, we have tested other starting points by adding a constant deviation $\Delta$ to the standard starting point. To avoid non-reproductibility, instead of using a random deviation, we built $\Delta=k \times(1,-1,1,-1, \ldots, 1,-1)$ with $k=$ $1,-10,100$. This makes it possible to check if the algorithms performs in a comparable way for different starting points and multiply by four the number of test cases. 


\subsubsection{Implementation}

The algorithms are written in MATLAB and the scripts have been run on a HPC cluster.

In order to compare the improvement of the new methods to the original PSB, the methods we implemented are PSB, gPSB Sym, gPSB MS, SUgPSB Sym and BFGS. SUgPSB MS is not tested as it is the same as gPSB MS. The initial approximation for the Hessian is $B_{0}=I$. An optional line search of the type MINPACK [30], with $\mu=0.01$ and $\eta=0.1$, is included within the algorithm.

The number $m$ of secant equations that are kept for the computation of the new estimate of the Hessian is of course a parameter. We used $m=2,4,8,16$ and for problems with dimension $n \geq 100$ also $m=64,256$. In order to avoid ill-conditioning, which arises when constituent vectors (secant equations) of the matrices $S_{i}$ and $Y_{i}$ become (nearly) linearly dependent, a QR filtering is applied [24].

Next to the environmental limitations like available memory on the cluster and time allowed for calculation (both having very little impact as stopping criteria in our experiment), the iteration is terminated when one of the following conditions is satisfied:

$-\left\|\nabla g\left(\mathbf{x}_{i}\right)\right\|_{2} \leq \gamma$

- After $n_{g}$ gradient function calls (this means that the potential function calls within the line search are not counted)

Multiple values for $\gamma$ and $n_{g}$ have been tested. In the following, the corresponding used values are indicated when presenting the results.

\subsubsection{Performance measurement}

Based on characteristic (d) in section 1, the efficiency of the formulas is compared based on the number of function calls or gradient function calls. The performance is compared based on Performance Profiles [1, 14].

For a given solver, the performance profile $\rho(\tau): \tau \in[1,+\infty[\rightarrow[0,1]$ is a nondecreasing, piecewise constant function, continuous from the right at each breakpoint. The probability that the solver will eventually solve the problem (without a limitation on the maximal amount of steps) is the value of $\rho(+\infty)$. On the other side, the probability that the solver will solve the problem more efficiently than the rest of the solvers is given by $\rho(1)$. On a graph, one can compare multiple algorithms. If the curve is higher, the algorithm solves more problems. If the curve is more on the left side, the algorithm solves the problems more quickly.

The metric of the efficiency can be defined in different ways: fewer function calls, less gradient calculation, less time... As mentioned in Section 1, characteristics (d), we use the number of gradient function calls as the metric. The results for function calls are however similar. 


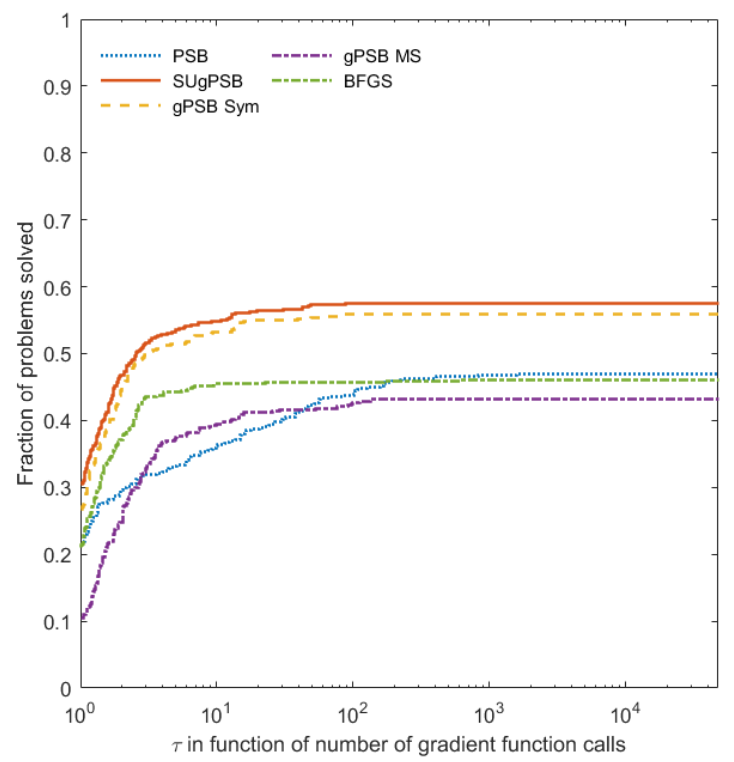

Fig. 3: Performance Profiles for $m=16, \gamma=10^{-6}, n_{g}=5 \times 10^{5}$ with line search.

As no known solutions are available for the problems in CUTEst, we use the fixed-cost approach mentioned in [1]. In the following analysis, we consider that an algorithm has converged to the correct solution if the value of the $\frac{f(\overline{\mathbf{X}})-f\left(\mathbf{X}^{*}\right)}{f\left(\mathbf{X}^{*}\right)}$ is less than $1 \%$, where $f(\overline{\mathbf{x}})$ is the final value of the objective function and $f\left(\mathbf{x}^{*}\right)$ the best known value of the problem (best solution within the tested algorithms). Combinations of problems and starting points for which none of the algorithm converged are excluded from the results.

\subsection{Results}

\subsubsection{Performance of SUgPSB}

Figures 3 and 4 show a comparison of the Performance Profile of PSB and the new formulas. We can observe multiple facts.

- Importance of symmetry above multisecant: First of all, we see a huge difference between gPSB Sym and gPSB MS. This tends to indicate that in our applications, the symmetric property is more important than the multisecant property.

- Improvement from multisecant: It is also clear that SUgPSB improves the efficiency of the PSB algorithm. The addition of pieces of information 


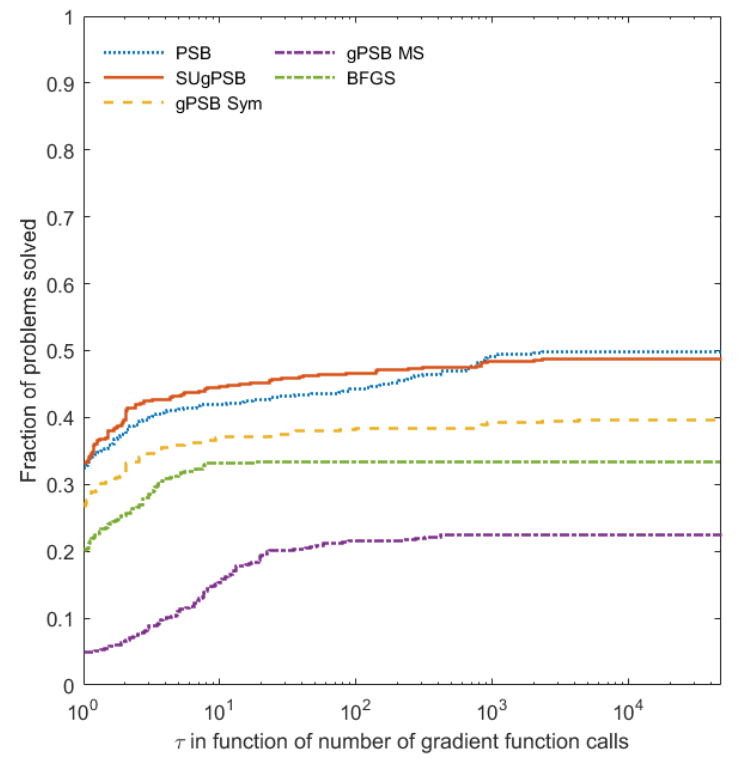

Fig. 4: Performance Profiles for $m=16, \gamma=10^{-5}, n_{g}=10^{5}$ without line search.

coming from extra secant equations makes the difference with PSB. SUgPSB even outperforms BFGS. To a lesser degree, adding the secant update property to gPSB Sym slightly improves the performance. We have also found empirically that the choice of $m$ has less influence on the performance of SUgPSB than on gPSB Sym. This seems to indicate that this Secant Update property contributes to the stability of the method.

- Increased impact of line search: By comparing the two figures, we highlight another interesting result. Whereas it has only limited impact on the results of the original PSB, the use of line search implies an important improvement on the performance of SUgPSB and gPSB Sym, increasing sensibly the number of problems that could be solved. This could indicate that the direction of the new step on the optimization path is better with those two formulas.

- Problem dependency: Next to those positive results, we must however mention that the performance profiles are dependent on the starting point. While for almost all tests the SUgPSB outperforms PSB, in one configuration (no line search, $k=1$ in the formula of deviation $\Delta$ in section 5.1 .1 ), it is not the case. This reminds us that performance can be problem dependent and reinforces our decision to test the algorithms with multiple starting points in order to multiply the number of test cases. 


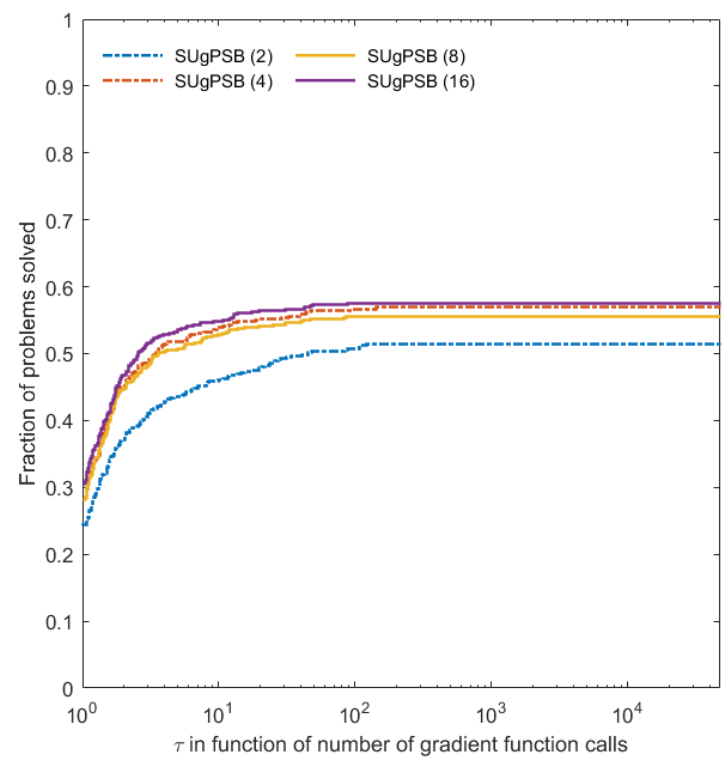

Fig. 5: Performance Profiles for $\gamma=10^{-6}, n_{g}=5 \times 10^{5}$ with line search. Value of $m$ is given between bracket in the legend of the graph.

\subsubsection{Impact of number of secant equations $m$}

The impact of the number of kept secant equations $m$ is very interesting. In Figure 5 , we see clearly that increasing the value of $m$ has a positive impact on the performance. This is the result we were expecting by creating SUgPSB. This shows that adding more secant equations helps to define a better estimate of the Hessian by using more pieces of information.

The improvement is however decreasing with the value of $m$. Every additional secant equation contributes less to the performance improvement. Moreover, depending on the problem, the effect of adding secant equations begins sooner or later to degrade the efficiency of the algorithm.

This is a consequence of the way the SUgPSB formula is built. The additional secant equations are considered as having the same importance as we tend to be the nearest to $K_{M S}=\left\{A \in \mathbb{R}^{n \times n}: A S_{i}=Y_{i}\right\}$ which encompasses every secant equation at once. Adding too old equations correspond to introducing obsolete information into the estimation of the Hessian. As there is no hierarchy between the secant equations within this formula, obsolete information offsets and eventually outweighs useful information. The quality of the new Hessian estimate is then degraded. 


\subsection{Further improvement}

It could be possible to mitigate the impact of too old secant equations by implementing some kind of forgetting mechanism or by adapting the formula in order to rank the used secant equations, and to reduce the relative weight of the oldest ones.

\section{Conclusion}

We wanted to improve the PSB Quasi-Newton method by developing a new formula for the estimate of the Hessian. The objective was to combine the characteristics of PSB (symmetric and satisfaction of last secant equation) with information coming from former secant equations from the optimization path (multisecant property).

In a first attempt, we have reproduced the results of Schnabel [37] proving the impossibility to combine the characteristics to build a gPSB formula for all cases. We provided an alternative proof which explained the apparent paradox of having fewer constraints than variables (an under-determined system of equations) and still be in the impossibility to find a solution. We also found two formulas that approximate the wanted gPSB formula by enforcing some properties and trying to approach as close as possible the properties that are not satisfied.

In a second phase, we have tried to improve the previously found approximated formulas by imposing the secant update property, which was missing in the previous approach. This leads to a new update formula, which we call SUgPSB.

Finally, the numerical experiments have shown that SUgPSB, used with a limited number of secant equations taken into account, clearly outperforms the existing PSB formula, the two versions of the gPSB we also developed, and even BFGS.

\section{References}

1. Beiranvand, V., Hare, W., Lucet, Y.: Best practices for comparing optimization algorithms. Optimization and Engineering 18(4), 815-848 (2017). URL https: //arxiv.org/pdf/1709.08242

2. Bertolazzi, E.: Quasi-Newton methods for minimization (2011). URL http://www . ing . unitn.it/ bertolaz/2-teaching/2011-2012/AA-2011-2012-OPTIM/ lezioni/slides-mQN.pdf

3. Boutet, N., Haelterman, R., Degroote, J.: Secant update version of quasi-Newton PSB with weighted multisecant equations. Computational Optimization and Applications pp. 1-26 (2020). URL https://biblio.ugent.be/publication/8644687/file/8644688

4. Boyd, S., Dattorro, J.: Alternating projections. EE392o, Stanford University (2003). URL https://pdfs.semanticscholar.org/1edo/ e86a12d31f1897b96b081489101a79da818a.pdf

5. Broyden, C.: On the discovery of the "good Broyden" method. Mathematical programming 87(2), 209-213 (2000). URL http://www.stat.uchicago.edu/ lekheng/ courses/31060s13/broyden.pdf 
6. Broyden, C.G.: A class of methods for solving nonlinear simultaneous equations. Mathematics of computation 19(92), 577-593 (1965). URL http://www.ams.org/ journals/mcom/1965-19-092/S0025-5718-1965-0198670-6/home.html

7. Broyden, C.G.: Quasi-Newton methods and their application to function minimisation. Mathematics of Computation 21(99), 368-381 (1967). URL https://pdfs. semanticscholar.org/46d5/fb4b4f686ba2479f0501e511d933a8cc057d.pdf

8. Cheney, W., Goldstein, A.A.: Proximity maps for convex sets. Proceedings of the American Mathematical Society 10(3), 448-450 (1959). URL https://pdfs. semanticscholar .org/02eb/6725b62ea0f85d338b26a11b3916b37f4997.pdf

9. Courrieu, P.: Fast computation of Moore-Penrose inverse matrices. arXiv preprint arXiv:0804.4809 (2008)

10. Degroote, J., Bathe, K.J., Vierendeels, J.: Performance of a new partitioned procedure versus a monolithic procedure in fluid-structure interaction. Computers \& Structures 87(11-12), 793-801 (2009). URL https://biblio.ugent.be/publication/533365/ file/533369.pdf

11. Degroote, J., Hojjat, M., Stavropoulou, E., Wüchner, R., Bletzinger, K.U.: Partitioned solution of an unsteady adjoint for strongly coupled fluid-structure interactions and application to parameter identification of a one-dimensional problem. Structural and Multidisciplinary Optimization 47(1), 77-94 (2013). URL https://biblio.ugent.be/ publication/3098489/file/3098584.pdf

12. Dennis, J., Walker, H.F.: Convergence theorems for least-change secant update methods. SIAM Journal on Numerical Analysis 18(6), 949-987 (1981). URL http://citeseerx. ist.psu.edu/viewdoc/download?doi=10.1.1.551.814\&rep=rep1\&type=pdf

13. Dennis Jr, J.E., Moré, J.J.: Quasi-Newton methods, motivation and theory. SIAM review 19(1), 46-89 (1977). URL https://hal.archives-ouvertes.fr/hal-01495720/ document

14. Dolan, E.D., Moré, J.J.: Benchmarking optimization software with performance profiles. Mathematical programming 91(2), 201-213 (2002). URL https://arxiv.org/pdf/cs/ 0102001

15. DuPré, A.M., Kass, S.: Distance and parallelism between flats in Rn. Linear Algebra and its Applications 171, 99-107 (1992). URL https://www.sciencedirect.com/science/ article/pii/0024379592902526/

16. Errico, R.M.: What is an adjoint model? Bulletin of the American Meteorological Society 78(11), 2577-2591 (1997). URL https://journals.ametsoc.org/doi/pdf/10. 1175/1520-0477 (1997) 078\%3C2577\%3AWIAAM\%3E2.0.CO\%3B2

17. Fang, H.r., Saad, Y.: Two classes of multisecant methods for nonlinear acceleration. Numerical Linear Algebra with Applications 16(3), 197-221 (2009). URL http: //www-users.cs.umn.edu/ saad/PDF/umsi-2007-100.pdf

18. Gould, N.I., Orban, D., Toint, P.L.: CUTEst: A constrained and unconstrained testing environment with safe threads for mathematical optimization. Computational Optimization and Applications 60(3), 545-557 (2015). URL ftp://www.hsl.rl.ac.uk/ $\mathrm{pub} / \mathrm{nimg} / \mathrm{pubs} /$ GoulOrbaToin15_coap.pdf

19. Gratton, S., Malmedy, V., Toint, P.L.: Quasi-Newton updates with weighted secant equations. Optimization Methods and Software 30(4), 748-755 (2015). URL http: //perso.fundp.ac.be/ phtoint/pubs/NTR-09-2013.pdf

20. Gratton, S., Toint, P.: Multi-secant equations, approximate invariant subspaces and multigrid optimization. Ph.D. thesis, tech. rep., Dept of Mathematics, FUNDP, Namur (B) (2007). URL http://perso.fundp.ac.be/ phtoint/pubs/TR07-11.pdf

21. Gross, J., Trenkler, G.: On the least squares distance between affine subspaces. Linear Algebra and its Applications 237, 269-276 (1996). URL https://www.sciencedirect. com/science/article/pii/0024379595006486/

22. Haelterman, R.: Analytical study of the least squares quasi-Newton method for interaction problems. Ph.D. thesis, Ghent University (2009). URL https://biblio. ugent.be/publication/720660

23. Haelterman, R., Bogaers, A., Degroote, J., Boutet, N.: Quasi-Newton methods for the acceleration of multi-physics codes. International Journal of Applied Mathematics 47(3) (2017). URL https://biblio.ugent.be/publication/8550797/file/8550798 
24. Haelterman, R., Bogaers, A.E., Scheufele, K., Uekermann, B., Mehl, M.: Improving the performance of the partitioned QN-ILS procedure for fluid-structure interaction problems: Filtering. Computers \& Structures 171, 9-17 (2016). URL https://researchspace.csir.co.za/dspace/bitstream/handle/10204/9109/ Haelterman_2016.pdf?sequence=1

25. Haelterman, R., Degroote, J., Van Heule, D., Vierendeels, J.: The quasi-Newton least squares method: A new and fast secant method analyzed for linear systems. SIAM Journal on numerical analysis 47(3), 2347-2368 (2009). URL https://www.researchgate.net/publication/235335399_The_Quasi-Newton_Least_ Squares_Method_A_New_and_Fast_Secant_Method_Analyzed_for_Linear_Systems

26. Khalfan, H.F., Byrd, R.H., Schnabel, R.B.: A theoretical and experimental study of the symmetric rank-one update. SIAM Journal on Optimization 3(1), 1-24 (1993). URL https://apps.dtic.mil/dtic/tr/fulltext/u2/a233965.pdf

27. Kim, D., Sra, S., Dhillon, I.S.: A new projected quasi-Newton approach for the nonnegative least squares problem. Tech. rep., Computer Science Department, University of Texas at Austin (2006). URL https://pdfs.semanticscholar.org/1e8c/ 118ad4e92c0927b19ec2bcb1ae8623aebde7.pdf

28. Mielczarek, D.: Minimal projections onto spaces of symmetric matrices. Univ. Iagel. Acta Math 44, 69-82 (2006). URL https://eudml.org/serve/128673/ accessibleLayeredPdf/0

29. Morales, J.L.: Variational quasi-Newton formulas for systems of nonlinear equations and optimization problems. (2008). URL http://users . eecs . northwestern. edu/ morales/ PSfiles/PSB.pdf

30. Moré, J.J., Thuente, D.J.: Line search algorithms with guaranteed sufficient decrease. ACM Transactions on Mathematical Software (TOMS) 20(3), 286-307 (1994). URL http://www.ii.uib.no/ Iennart/drgrad/More1994.pdf

31. Pang, C.J.: Accelerating the alternating projection algorithm for the case of affine subspaces using supporting hyperplanes. Linear Algebra and its Applications 469, 419-439 (2015). URL https://www.sciencedirect.com/science/article/pii/ S0024379514007708

32. Plessix, R.E.: A review of the adjoint-state method for computing the gradient of a functional with geophysical applications. Geophysical Journal International 167(2), 495-503 (2006). URL https://academic.oup.com/gji/article-pdf/167/2/ 495/1492368/167-2-495.pdf

33. Powell, M.: Beyond symmetric Broyden for updating quadratic models in minimization without derivatives. Mathematical Programming 138(1-2), 475-500 (2013). URL http://www6.cityu.edu.hk/rcms/publications/preprint28.pdf

34. Powell, M.J.: A new algorithm for unconstrained optimization. In: Nonlinear Programming, pp. 31-65. Elsevier (1970). URL https://www.sciencedirect.com/ science/article/pii/B9780125970501500063

35. Rheinboldt, W.C.: Quasi-Newton methods. Lecture Notes, TU Munich (2000). URL https://www-m2.ma.tum.de/foswiki/pub/M2/Allgemeines/SemWs09/quasi-newt.pdf

36. Scheufele, K., Mehl, M.: Robust Multisecant Quasi-Newton Variants for Parallel Fluid-Structure Simulations - and Other Multiphysics Applications. SIAM Journal on Scientific Computing 39(5), S404-S433 (2017). URL ftp://ftp.informatik.uni-stuttgart.de/pub/library/ncstrl.ustuttgart_fi/ ART-2017-11/ART-2017-11.pdf

37. Schnabel, R.B.: Quasi-Newton methods using multiple secant equations. Tech. rep., DTIC Document (1983). URL http://www.dtic.mil/cgi-bin/GetTRDoc?AD=ADA131444 


\section{Appendices}

\section{A Preliminary lemmas}

In the following proofs, we will apply the following simplifications (and their transposed version). Let $X \in \mathbb{R}^{n \times m}, Y \in \mathbb{R}^{n \times m}$, and $\mathbf{x}$ the last column of $X$ (same for $\mathbf{y}$ and $Y$ ), we give here some interesting results.

Lemma A.1

$$
\begin{gathered}
X X^{+} \boldsymbol{x}=\boldsymbol{x} \\
\boldsymbol{x}^{T}\left(X^{+}\right)^{T} X^{T}=\boldsymbol{x}^{T}
\end{gathered}
$$

Proof $X X^{+} \mathbf{x}$ is the last column of $X X^{+} X$, but $X X^{+} X=X$. So $X X^{+} \mathbf{x}$ is the last column of $X$ which is $\mathbf{x}$. The second form is simply the transposed expression.

Lemma A.2

$$
\begin{gathered}
\boldsymbol{x}^{T} X X^{+}=\boldsymbol{x}^{T} \\
\left(X^{+}\right)^{T} X^{T} \boldsymbol{x}=\boldsymbol{x}
\end{gathered}
$$

Proof $\mathbf{x}^{T} X X^{+}$is the last row of $X^{T} X X^{+}$. But $X^{T} X X^{+}=X^{T} X\left(X^{T} X\right)^{-1} X^{T}=X^{T}$. So $\mathbf{x}^{T} X X^{+}$is the last row of $X^{T}$ which is $\mathbf{x}^{T}$. The second form is simply the transposed expression.

Lemma A.3

$$
\begin{gathered}
Y X^{+} \boldsymbol{x}=\mathbf{y} \\
\boldsymbol{x}^{T}\left(X^{+}\right)^{T} Y^{T}=\mathbf{y}^{T}
\end{gathered}
$$

Proof $Y X^{+} \mathbf{x}$ is the last column of $Y X^{+} X$. But $Y X^{+} X=Y$. So $Y X^{+} \mathbf{x}$ is the last column of $Y$ which is $\mathbf{y}$. The second form is simply the transposed expression.

\section{B Alternating Projections applied on PSB}

B.1 Closed convex sets

Here are the proofs that the sets used in Section 3.2 are closed and convex.

Lemma B.1 The set of symmetric matrices is a closed convex set.

Proof The set of symmetric matrices is a vector subspace. So it is closed and convex.

Lemma B.2 The set of multisecant matrices is a closed convex set.

Proof The set of multisecant matrices is the set of matrices A such that $A \mathbf{x}=\mathbf{b}$. This is an affine space. So it is closed and convex. 
B.2 Projection on the set of multisecant matrices

The formula for the projection on $K_{M S}$ is (3.2):

$$
{ }_{i+1} B={ }_{i} B+\left(Y_{i}-{ }_{i} B S_{i}\right)\left(S_{i}^{T} S_{i}\right)^{-1} S_{i}^{T}
$$

Proof For more readability and to avoid to handle too many subscripts, we lightly change the notation in this development. We note:

$-{ }_{i+1} B \equiv A$
$-{ }_{i} B \equiv B$

- We omit the subscript $i$ for $S$ and $Y$.

- $j, k$ the scalar coordinates within a vector or a matrix ( $j$-th row, $k$-th column).

We start with the following optimization problem:

$$
\begin{gathered}
\arg \min _{A} \frac{1}{2}\|A-B\|_{F r}^{2} \\
\text { such that } A S-Y=0
\end{gathered}
$$

We take the Lagrangian of the system:

$$
\mathcal{L}(A, \Lambda)=\frac{1}{2}\|A-B\|_{F r}^{2}+\sum_{j, k} \Lambda_{j, k}\left(\sum_{l} A_{j, l} S_{l, k}-Y_{j, k}\right)
$$

We now take the partial derivative in function of $A_{j, k}$. We first note that:

$$
\begin{aligned}
\frac{\partial}{\partial A_{j, k}} \sum_{j, k} \Lambda_{j, k}\left(\sum_{l} A_{j, l} S_{l, k}-Y_{j, k}\right) & =\frac{\partial}{\partial A_{j, k}} \sum_{j, k, l} \Lambda_{j, k} A_{j, l} S_{l, k} \\
& =\frac{\partial}{\partial A_{j, k}} \sum_{j, l, k} \Lambda_{j, l} A_{j, k} S_{k, l} \\
& =\sum_{l} \Lambda_{j, l} S_{k, l} \\
& =\left(\Lambda S^{T}\right)_{j, k}
\end{aligned}
$$

We find:

$$
\frac{\partial \mathcal{L}}{\partial A_{j, k}}=A_{j, k}-B_{j, k}+\left(\Lambda S^{T}\right)_{j, k}=0
$$

The system can thus be written

as:

$$
\begin{cases}A-B+\Lambda S^{T} & =0 \\ A S-Y & =0\end{cases}
$$

Putting (B.1) into (B.2), we find:

$$
\begin{aligned}
A S-Y & =0 \\
B S-\Lambda S^{T} S-Y & =0 \\
\Lambda S^{T} S & =B S-Y \\
\Lambda & =(B S-Y)\left(S^{T} S\right)^{-1}
\end{aligned}
$$

Putting this back into (B.1), we have a new update formula:

$$
\begin{aligned}
A & =B+(Y-B S)\left(S^{T} S\right)^{-1} S^{T} \\
B_{i+1} & =B_{i}+\left(Y_{i}-B_{i} S_{i}\right)\left(S_{i}^{T} S_{i}\right)^{-1} S_{i}^{T}
\end{aligned}
$$


B.3 generalized PSB

Proof of Theorem 1 and Theorem 2.

Proof As explained in section 3.1, we use alternating projection. We project alternatively on the subspace of multisecant matrices $\left(K_{M S}\right)$ and on the subspace of symmetric matrices $\left(K_{\text {Sym }}\right)$ :

$-K_{M S}$ : Defined in (3.2) for the projection on the set of multisecant matrices. We call the projection ${ }_{j} B$.

- $K_{\text {Sym }}$ : Equation (3.1) for the projection on the set of symmetric matrices. We call the projection ${ }_{j} \bar{B}$.

We recall that $\left(S^{T} S\right)^{-1} S^{T}=S^{+}$(Moore-Penrose pseudoinverse). We start with ${ }_{0} B$ and we develop:

$$
\begin{aligned}
{ }_{1} B & ={ }_{0} B-{ }_{0} B S S^{+}+Y S^{+} \\
{ }_{1} \bar{B} & =\frac{1 B}{2}+\frac{{ }_{1} B^{T}}{2} \\
& =\frac{{ }_{0} B^{T}}{2}+\frac{{ }_{0} B}{2}-\frac{{ }_{0} B S S^{+}}{2}-\frac{\left(S^{+}\right)^{T} S^{T}{ }_{0} B^{T}}{2}+\frac{\left(S^{+}\right)^{T} Y^{T}}{2}+\frac{Y S^{+}}{2}
\end{aligned}
$$

After those two first projections, we go on:

$$
\begin{aligned}
{ }_{2} B= & { }_{1} B-{ }_{1} B S S^{+}+Y S^{+} \\
= & \frac{{ }_{0} B^{T}}{2}-\frac{{ }_{0} B^{T} S S^{+}}{2}+\frac{{ }_{0} B}{2}-\frac{{ }_{0} B S S^{+}}{2}-\frac{\left(S^{+}\right)^{T} S^{T}{ }_{0} B^{T}}{2} \\
& +\frac{\left(S^{+}\right)^{T} S^{T}{ }_{0} B^{T} S S^{+}}{2}+\frac{\left(S^{+}\right)^{T} Y^{T}}{2}-\frac{\left(S^{+}\right)^{T} Y^{T} S S^{+}}{2}+Y S^{+} \\
{ }_{2} \bar{B}= & \frac{1}{2}\left({ }_{2} B+{ }_{2} B^{T}\right) \\
= & \frac{{ }_{0} B^{T}}{2}-\frac{1}{4}{ }_{0} B^{T} S S^{+}+\frac{{ }_{0} B}{2}-\frac{{ }_{0} B S S^{+}}{2} \\
& -\frac{\left(S^{+}\right)^{T} S^{T}{ }_{0} B^{T}}{2}+\frac{1}{4}\left(S^{+}\right)^{T} S^{T}{ }_{0} B^{T} S S^{+}-\frac{1}{4}\left(S^{+}\right)^{T} S^{T}{ }_{0} B+\frac{1}{4}\left(S^{+}\right)^{T} S^{T}{ }_{0} B S S^{+} \\
& -\frac{1}{4}\left(S^{+}\right)^{T} S^{T} Y S^{+}+\frac{3}{4}\left(S^{+}\right)^{T} Y^{T}-\frac{1}{4}\left(S^{+}\right)^{T} Y^{T} S S^{+}+\frac{3}{4} Y S^{+} \\
{ }_{3} B= & \frac{{ }_{0} B^{T}}{2}-\frac{{ }_{0} B^{T} S S^{+}}{2}+\frac{{ }_{0} B}{2}-\frac{{ }_{0} B S S^{+}}{2}-\frac{\left(S^{+}\right)^{T} S^{T}{ }_{0} B^{T}}{2}+\frac{\left(S^{+}\right)^{T} S^{T}{ }_{0} B^{T} S S^{+}}{2} \\
& -\frac{1}{4}\left(S^{+}\right)^{T} S^{T}{ }_{0} B+\frac{1}{4}\left(S^{+}\right)^{T} S^{T}{ }_{0} B S S^{+}+\frac{3}{4}\left(S^{+}\right)^{T} Y^{T}-\frac{3}{4}\left(S^{+}\right)^{T} Y^{T} S S^{+}+Y S^{+}
\end{aligned}
$$

We define:

$$
\begin{aligned}
{ }_{j} B= & \frac{{ }_{0} B^{T}}{2}-\frac{{ }_{0} B^{T} S S^{+}}{2}+\frac{{ }_{0} B}{2}-\frac{{ }_{0} B S S^{+}}{2}-\frac{\left(S^{+}\right)^{T} S^{T}{ }_{0} B^{T}}{2}+\frac{\left(S^{+}\right)^{T} S^{T}{ }_{0} B^{T} S S^{+}}{2} \\
& -\frac{2^{j-2}-1}{2^{j-1}}\left(S^{+}\right)^{T} S^{T}{ }_{0} B+\frac{2^{j-2}-1}{2^{j-1}}\left(S^{+}\right)^{T} S^{T}{ }_{0} B S S^{+} \\
& +\frac{2^{j-1}-1}{2^{j-1}}\left(S^{+}\right)^{T} Y^{T}-\frac{2^{j-1}-1}{2^{j-1}}\left(S^{+}\right)^{T} Y^{T} S S^{+}+Y S^{+} \\
{ }_{j} \bar{B}= & \frac{0 B^{T}}{2}-\frac{2^{j-1}-1}{2^{j}}{ }_{0} B^{T} S S^{+}+\frac{{ }_{0} B}{2}-\frac{{ }_{0} B S S^{+}}{2}-\frac{\left(S^{+}\right)^{T} S^{T}{ }_{0} B^{T}}{2} \\
& +\frac{2^{j-1}-1}{2^{j}}\left(S^{+}\right)^{T} S^{T}{ }_{0} B^{T} S S^{+}-\frac{2^{j-1}-1}{2^{j}}\left(S^{+}\right)^{T} S^{T}{ }_{0} B+\frac{2^{j-1}-1}{2^{j}}\left(S^{+}\right)^{T} S^{T}{ }_{0} B S S^{+} \\
& -\frac{2^{j-1}-1}{2^{j}}\left(S^{+}\right)^{T} S^{T} Y S^{+}+\frac{2^{j}-1}{2^{j}}\left(S^{+}\right)^{T} Y^{T}-\frac{2^{j-1}-1}{2^{j}}\left(S^{+}\right)^{T} Y^{T} S S^{+}+\frac{2^{j}-1}{2^{j}} Y S^{+}
\end{aligned}
$$


We can easily see that, when $j=2,{ }_{j} \bar{B}$ corresponds to ${ }_{2} \bar{B}$ and, when $j=3,{ }_{j} B$ corresponds to ${ }_{3} B$. We also easily check that ${ }_{j} \bar{B}$ is the projection of ${ }_{j} B$ using equation (3.1), and that projecting ${ }_{j} \bar{B}$ with (3.2) gives ${ }_{j+1} B$.

Finally, taking the limit to infinity, we see that the sequences converge to two different formulas. On one side, we have $\lim _{j \rightarrow \infty} j \bar{B}$, the symmetric formula closest to the space of matrices satisfying multiple secant equations: gPSB Sym. This proves Theorem 1.

The second formula, $\lim _{j \rightarrow \infty} B$, gives the matrix satisfying multiple secant equations and being the closest to the symmetric matrix: gPSB MS. This proves Theroem 2.

\section{B.4 Existence conditions of gPSB}

\section{B.4.1 Theorem 3}

We give here a proof to Theorem 3, which is an alternating proof of the theorem of impossibility of existence of gPSB of Schnabel [37].

Proof We are looking for $B \in \mathbb{R}^{n \times n}: B S=Y$ and $B=B^{T}$ with $S$ and $Y \in \mathbb{R}^{n \times m}$.

Step 1: construction of $S^{\text {syst }}$

We will first construct a matrix that we will call $S^{\text {syst }}$. Therefore, using the underscript $X_{*, k}$ as the $k$-th column of the matrix $\mathrm{S}$, we notice that $B \mathbf{s}_{*, 1}=\mathbf{y} *, 1$ can be expressed as $S^{1} \mathbf{b}=\mathbf{y}_{*, 1}$ where

$-\mathbf{b}=\operatorname{vec}(B)$, a column vector containing every element of $B$.

- $\mathbf{s}_{*, 1}$ being a column vector containing the first column of $S . \mathbf{y}_{*, 1}$ the first column of $Y$.

- $S^{1}$ a block diagonal $n \times n^{2}$ matrix containing the line vector $\mathbf{s}_{*, 1}^{T}$ in each block of the diagonal.

We create $S^{i}$ and $\mathbf{y}_{*, i}$ in the same way for the following columns of $S$ and $Y$.

We now express the symmetry condition in the same form: $\Sigma \mathbf{b}=\mathbf{0}$. $\Sigma$ is a $\frac{n(n-1)}{2} \times n^{2}$ matrix. For each couple $\left\{b_{i j}, b_{j i}\right\}(1 \leq i \leq n-1, i+1 \leq j \leq n)$, we have a line containing 0 everywhere except for the place corresponding to $b_{i j}$ and $b_{j i}$ where the value is respectively 1 and -1 .

Applying the 2 conditions $B S=Y$ and $B=B^{T}$ together, we have thus:

$$
\begin{aligned}
{\left[\left(S^{1}\right)^{T}\left|\left(S^{2}\right)^{T}\right| \ldots\left|\left(S^{m}\right)^{T}\right| \Sigma\right]^{T} \mathbf{b} } & =\left[\mathbf{y}_{*, 1}^{T}\left|\mathbf{y}_{*, 2}^{T}\right| \ldots\left|\mathbf{y}_{*, m}^{T}\right| 0\right]^{T} \\
S^{\text {syst }} \mathbf{b} & =\mathbf{y}_{i}
\end{aligned}
$$

Here we used the symbol $\left[X_{1} \mid X_{2}\right]$ to indicate the concatenation of column vectors next to each other. See illustration in Example 1.

Example 1 For instance, for $n=3$ and $m=2$, we have:

$$
S^{\text {syst }}=\left[\begin{array}{ccccccccc}
s_{11} & s_{21} & s_{31} & 0 & 0 & 0 & 0 & 0 & 0 \\
0 & 0 & 0 & s_{11} & s_{21} & s_{31} & 0 & 0 & 0 \\
0 & 0 & 0 & 0 & 0 & 0 & s_{11} & s_{21} & s_{31} \\
s_{12} & s_{22} & s_{32} & 0 & 0 & 0 & 0 & 0 & 0 \\
0 & 0 & 0 & s_{12} & s_{22} & s_{32} & 0 & 0 & 0 \\
0 & 0 & 0 & 0 & 0 & 0 & s_{12} & s_{22} & s_{32} \\
0 & 1 & 0 & -1 & 0 & 0 & 0 & 0 & 0 \\
0 & 0 & 1 & 0 & 0 & 0 & -1 & 0 & 0 \\
0 & 0 & 0 & 0 & 0 & 1 & 0 & -1 & 0
\end{array}\right]
$$

Step 2: Linear dependence for $m=2$

Let's build $S^{\text {syst }}$ for $m=2$. We apply then the following linear combination: 
- We multiply the $n$ first rows by the coefficients of $\mathbf{s}_{*, 2}$.

- We multiply the $n$ following rows by the coefficient of $-\mathbf{s}_{*, 1}$.

- For the column corresponding to $b_{i i}$, the linear combination of the rows is then equal to zero. There are only zeroes in the rows of $\Sigma$ for these columns.

- For the columns corresponding to $b_{i j}$ with $i \neq j$, the linear combination on the $2 n$ first rows gives a value $\delta_{i j}$. We notice that $\delta_{i j}=-\delta_{j i}$. But for these columns, there is a single row of $\Sigma$ having 1 in the column corresponding to $b_{i j}(i<j),-1$ in the column of $b_{j i}$ and 0 everywhere else. No other row of $\Sigma$ has a non-zero value under the column of $b_{i j}$ or $b_{j i}$. So we multiply that rows by $-\delta_{i j}$ and the linear combination gives 0 for those columns, too.

See Example 2 for an illustration.

Applying this linear combination to the matrix leads to a row containing only zeroes. So there is at least one linear dependency between the rows of $S^{\text {syst }}$. The rank of matrix $S^{\text {syst }}$ is at least one less than its number of lines.

Example 2 For our example with $n=3$, we associate the following coefficients to the successive row:

$$
\left\{s_{12}, s_{22}, s_{32},-s_{11},-s_{21},-s_{31}, s_{22} s_{11}-s_{21} s_{12}, s_{32} s_{11}-s_{31} s_{12}, s_{32} s_{21}-s_{31} s_{22}\right\}
$$

Using this coefficient to define a linear combination of the rows, we can easily check that this combination leads to $\mathbf{0}$.

Step 3: Existence conditions for $m=2$

$\Rightarrow$ If $B S=Y$ has a solution, then $S^{\text {syst }} \mathbf{b}=\mathbf{y}_{i}$ has a solution, too. In this case, the linear combination of the lines of matrix $S^{\text {syst }}$ also holds for $\mathbf{y}_{i}$. This linear combination applied to $\mathbf{y}_{i}$ gives:

$$
\mathbf{s}_{*, 2}^{T} \mathbf{y}_{*, 1}+\left(-\mathbf{s}_{*, 1}^{T}\right) \mathbf{y}_{*, 2}+0=0
$$

$\Leftarrow$ On the other side, if $\mathbf{s}_{*, 2}^{T} \mathbf{y}_{*, 1}+\left(-\mathbf{s}_{*, 1}^{T}\right) \mathbf{y}_{*, 2}=0$, then applying the linear combination on $\mathbf{y}_{i}$ gives 0 . The linear combination of $S^{\text {syst }}$ also holds for $\mathbf{y}_{i}$, so $S^{\text {syst }} \mathbf{b}=\mathbf{y}_{i}$ has a solution which is then also the case for $B S=Y$

Step 4: Conditions for $m>2$

Extending to higher values of $m$, for each pair $i, j$ of the columns of $S$ and $Y$, we have a solution if and only if $\mathbf{s}_{*, i}^{T} \mathbf{y}_{*, j}=\mathbf{s}_{*, j}^{T} \mathbf{y}_{*, i}=\mathbf{y}_{*, i}^{T} \mathbf{s}_{*, j}$. For the second equality we used the fact that the transpose of a scalar is the scalar itself. This is equivalent to $\left[S^{T} Y\right]_{i, j}=\left[Y^{T} S\right]_{i, j}$ which leads to $S^{T} Y=Y^{T} S$.

\section{B.4.2 Theorem 4}

We give here the proof of Theorem 4 .

Proof Step 0: Linear dependencies for $m=2$

This has been shown above in the alternative proof of Theorem 3. By reasoning column by column, we have found one single linear combination involving all the rows.

Step 1: Linear dependencies for $m=3$

For $m=3$, we have $S_{3}^{\text {syst }}=\left[S_{1}^{T}\left|S_{2}^{T}\right| S_{3}^{T} \mid \Sigma\right]^{T}$ (see previous demo for details about the construction of this matrix). Thanks to step 0 , we know that we have 3 linear combinations (one for each couple of columns of the matrix $S$ ). However, those linear combinations can be equivalent, as one of them could be a combination of the other two. We have to prove that those combinations are different. We will proceed by contradiction: say that such a linear combination exists and prove that this is only possible if $S$ is not full-rank. We define $S_{2}^{\text {syst }}=\left[S_{1}^{T}\left|S_{2}^{T}\right| \Sigma\right]^{T}$.

The following facts are noted: 
1. By hypothesis, we have $n \geq 3$.

2. The dimension of $S_{2}^{\text {syst }}$ is $\frac{n(n+3)}{2} \times n^{2}$. The number of rows is less or equal to the number of columns.

3. As we have proven that, in $S_{2}^{\text {syst }}$, there is only one linear combination involving every row, we can take a subset of $\frac{n(n+3)}{2}-1$ rows without losing information nor reducing the rank of the system.

4. There are at least $n+1$ independent rows in $S_{2}^{\text {syst }}$. We have indeed $n$ independent rows in $S_{1}$ and at least one extra independent row in $S_{2}$ because $S$ is full-rank. Therefore, the dimension of the span of its rows is at least $n+1$.

5. The dimension of the span of the rows of $S_{3}$ is at most $n$.

This leads us to the following conclusion: If the span of $S_{3}$ is included in the span of $S_{2}^{\text {syst }}$, then there is only one linear combination in $S_{3}^{\text {syst }}$. Otherwise, there are 3 distinct linear combinations.

For the proof by contradiction, we assume that the span of $S_{3}$ is included in the span of $S_{2}^{\text {syst }}$. Then, for each row of $S_{3}$, there exists a linear combination of the rows of $S_{2}^{\text {syst }}$ that is equal to it. Thanks to point 3 above, we know that we can remove one row of $S_{2}^{\text {syst }}$. We start thus by replacing the first row of $S_{3}$ (See Example 3).

Example 3 For the case $n=3$, we have then:

$$
\left[\begin{array}{ccccccccc}
s_{13} & s_{23} & s_{33} & 0 & 0 & 0 & 0 & 0 & 0 \\
0 & 0 & 0 & s_{11} & s_{21} & s_{31} & 0 & 0 & 0 \\
0 & 0 & 0 & 0 & 0 & 0 & s_{11} & s_{21} & s_{31} \\
s_{12} & s_{22} & s_{32} & 0 & 0 & 0 & 0 & 0 & 0 \\
0 & 0 & 0 & s_{12} & s_{22} & s_{32} & 0 & 0 & 0 \\
0 & 0 & 0 & 0 & 0 & 0 & s_{12} & s_{22} & s_{32} \\
0 & 1 & 0 & -1 & 0 & 0 & 0 & 0 & 0 \\
0 & 0 & 1 & 0 & 0 & 0 & -1 & 0 & 0 \\
0 & 0 & 0 & 0 & 0 & 1 & 0 & -1 & 0
\end{array}\right]
$$

We apply then the following linear combination:

- We multiply the $n$ first rows by the coefficients of $\mathbf{s}_{*, 2}$.

- We multiply the $n+1$-th row by $-\mathbf{s}_{1,3}$.

- We multiply the $n-1$ following rows by the coefficient of $-\mathbf{s}_{*, 1}$.

- For the column corresponding to $b_{i i}$, the linear combination of the rows is then equal to zero (there are only zeroes in the rows of $\Sigma$ for these columns).

- For the columns corresponding to $b_{i j}$ with $i \neq j$ and $i, j \neq 1$, the linear combination on the $2 n$ first rows gives a value $\delta_{i j}$

- We notice that $\delta_{i j}=-\delta_{j i}$.

- But for these columns, there is a single row of $\Sigma$ having 1 in the column corresponding to $b_{i j}(i<j),-1$ in the column of $b_{j i}$ and 0 everywhere else.

- No other row of $\Sigma$ has a non-zero value under the column of $b_{i j}$ or $b_{j i}$.

- So we multiply those rows by $-\delta_{i j}$ and the linear combination gives 0 for those columns, too.

- For the columns corresponding to $b_{1 i}$ and $b_{i 1}$ with $i \neq 1$, we should also have $\delta_{1 i}=-\delta_{i 1}$, but the equation is not automatically satisfied. This gives us $n-1$ equations (for $i=2, \ldots, n-1)$ :

$$
\mathbf{s}_{1,2} \mathbf{s}_{i, 3}-\mathbf{s}_{i, 2} \mathbf{s}_{1,3}=\mathbf{s}_{1,2} \mathbf{s}_{i, 1}-\mathbf{s}_{i, 2} \mathbf{s}_{1,1}
$$

Applying the same reasoning but after replacing the $n+1$-th row of $S_{2}^{\text {syst }}$ by the first row of $S_{3}$ leads to another $n+1$ equations:

$$
\mathbf{s}_{1,1} \mathbf{s}_{i, 3}-\mathbf{s}_{i, 1} \mathbf{s}_{1,3}=\mathbf{s}_{1,1} \mathbf{s}_{i, 2}-\mathbf{s}_{i, 1} \mathbf{s}_{1,2}
$$


Combining (B.3) and (B.4) leads to:

$$
\begin{gathered}
\mathbf{s}_{1,2} \mathbf{s}_{i, 3}-\mathbf{s}_{i, 2} \mathbf{s}_{1,3}=\mathbf{s}_{i, 1} \mathbf{s}_{1,3}-\mathbf{s}_{1,1} \mathbf{s}_{i, 3} \\
\frac{\mathbf{s}_{1,3}}{\mathbf{s}_{i, 3}}=\frac{\mathbf{s}_{1,2}+\mathbf{s}_{1,1}}{\mathbf{s}_{i, 2}+\mathbf{s}_{i, 1}}
\end{gathered}
$$

We can now apply the same process but starting with successively replacing the $i$-th and $n+i$-th rows of $S_{2}^{\text {syst }}$ by the $i$-th row of $S_{3}$, for $i=2, \ldots, n$. This leads to the general equations:

$$
\frac{\mathbf{s}_{i, 3}}{\mathbf{s}_{i, 2}+\mathbf{s}_{i, 1}}=k \text { for } i=1, \ldots, n \text { where } k \text { is a constant }
$$

The consequence is $\mathbf{s}_{*, 3}=k\left(\mathbf{s}_{*, 1}+\mathbf{s}_{*, 2}\right) . S$ is then not full-rank which is in contradiction with our hypothesis. So the span of $S_{3}$ is not included in the span of $S_{2}^{\text {syst }}$ and, for $m=3$, we have 3 distinct linear combinations.

Step 2: Linear dependencies for $m>3$

The proof extends to higher values of $m$ by applying Step 3 on every combination of 3 submatrices $S_{i}$. So each additional column of $S$ adds one new linear combination with every other column. In general, for $n$ and $m$ given, we have a system of $m n+\frac{n^{2}-n}{2}$ equations with $n^{2}$ variables, but the rank of the matrix is only $m n+\frac{n^{2}-n}{2}-\frac{m^{2}-m}{2}$.

\section{SUgPSB}

\section{Alternating Projections applied on SUgPSB}

\section{D.1 Closed convex sets}

Here are the proofs that the sets used in Section 4.1 are closed and convex.

Lemma D.1 The set of symmetric matrices satisfying the last secant equation is a closed convex set.

Proof This set is in fact a projection on the intersection of the set $K_{S y m}$ and $K_{S U}$, the latter being a special case of $K_{M S}$ (one secant equation instead of multiple secant equations).

In Lemma B.1, we have already proved that $K_{S y m}$ is closed and convex.

By application of Lemma B.2 on $K_{S U}$ where we only take one column of $S$ and $Y$, we prove that $K_{S U}$ is a closed convex set.

As both sets $\left(K_{S y m}\right.$ and $\left.K_{S U}\right)$ are closed and convex, their intersection $K_{S y m S U}$ is closed and convex.

Lemma D.2 Within the set of multisecant matrices for given secant equations, the subset of matrices being the nearest to be a symmetric matrix is a closed convex set.

Proof The set of multisecant matrices $\left(K_{M S}\right)$ is an affine space. The set of symmetric matrices $\left(K_{S y m}\right)$ is a vector subspace, which is a special case of an affine subspace where the origin point is null.

The end space $E_{1}$ as defined in [15,21], is the set of points defined as $E_{1}:=$ $\left\{\mathbf{x} \in L_{1}: d\left(\mathbf{x}, L_{2}\right)=d\left(L_{1}, L_{2}\right)\right\}$, where $L_{1}$ and $L_{2}$ are affine subspaces and $d$ is the distance between a point and an affine subspace or between two affine subspaces. In Theorem 2 of [15], it is shown that $\mathbf{x} \in E_{1}$ solves an equation of the form $A \mathbf{x}=\mathbf{b}$, so $E_{1}$ is an affine space. Applying this with $K_{M S}$ as $L_{1}$ and $K_{S y m}$ as $L_{2}$, we prove that $K_{M S \triangleright S y m}$ is an affine subspace, so it is closed and convex. 
D.2 PSB non symmetric start

We prove here the formula (4.1).

Theorem 9 (PSB - Direct Form from non symmetric matrix) Let $B_{i} \in \mathbb{R}^{n \times n}, \mathbf{y}_{i}$ and $\mathbf{s}_{i} \in \mathbb{R}^{n \times 1}$ and $S_{i}$ full-rank. Let $B_{i+1}$ such that:

$-B_{i+1} \mathbf{s}_{i}=\mathbf{y}_{i}$

- $\left\|B_{i+1}-B_{i}\right\|_{F}$ is minimal

Then, $B_{i+1}$ is given by:

$$
B_{i+1}=\bar{B}_{i}+\frac{\overline{\mathbf{w}}_{i} \mathbf{s}_{i}^{T}}{\mathbf{s}_{i}^{T} \mathbf{s}_{i}}+\frac{\mathbf{s}_{i} \overline{\mathbf{w}}_{i}^{T}}{\mathbf{s}_{i}^{T} \mathbf{s}_{i}}-\frac{\overline{\mathbf{w}}_{i}^{T} \mathbf{s}_{i}}{\left(\mathbf{s}_{i}^{T} \mathbf{s}_{i}\right)^{2}} \mathbf{s}_{i} \mathbf{s}_{i}^{T}
$$

With

- $\bar{B}_{i}=\frac{B_{i}+B_{i}^{T}}{2}$

$-\overline{\mathbf{w}}_{i}=\mathbf{y}_{i} \stackrel{2}{-} \bar{B}_{i} \mathbf{s}_{i}$

Proof We will apply the method of alternating projection with:

- $K_{S y m}$ : equation (3.1) for the symmetrical projection, giving ${ }_{j} \bar{B}$

- $K_{S U}$ : Broyden "good" $[5,6]$ for the secant update projection, giving ${ }_{j} B$

Let's start with ${ }_{0} B$. We first project onto the set of symmetric matrices. For more readability and as we work within one step of the Quasi-Newton process, we omit the subscripts $i$ which refers to the step of the Quasi-Newton process. We have:

$$
\begin{aligned}
{ }_{0} \bar{B} & =\frac{{ }_{0} B+{ }_{0} B^{T}}{2} \\
{ }_{0} \overline{\mathbf{w}}_{i} & =\mathbf{y}_{i}-{ }_{0} \bar{B} s \\
& =\mathbf{y}_{i}-\frac{{ }_{0} B s+{ }_{0} B^{T} s}{2} \\
{ }_{1} B & ={ }_{0} \bar{B}+\frac{{ }_{0} \overline{\mathbf{w}}_{i} \mathbf{s}_{i}^{T}}{\mathbf{s}_{i}^{T} \mathbf{s}_{i}}+\frac{\mathbf{s}_{i 0} \overline{\mathbf{w}}_{i}^{T}}{\mathbf{s}_{i}^{T} \mathbf{s}_{i}}-\frac{{ }_{0} \overline{\mathbf{w}}_{i}^{T} \mathbf{s}_{i}}{\left(\mathbf{s}_{i}^{T} \mathbf{s}_{i}\right)^{2}} \mathbf{s}_{i} \mathbf{s}_{i}^{T}
\end{aligned}
$$

We should now project on symmetric matrices again. But ${ }_{1} B$ is already symmetric. So the alternating projection has already converged.

\section{D.3 SUgPSB Sym \& SUgPSB MS}

Proof of Theorem 5 and 6 .

Proof We project alternatively on the 2 sets: $K_{M S \triangleright S y m}$ and $K_{S y m S U}$.

$$
\begin{aligned}
{ }_{1} B_{M S \triangleright S y m}= & B+\left(S^{+}\right)^{T} Y^{T}+Y S^{+}-B S S^{+}-\left(S^{+}\right)^{T} S^{T} B^{T} \\
& -\left(S^{+}\right)^{T} Y^{T} S S^{+}+\left(S^{+}\right)^{T} S^{T} B^{T} S S^{+} \\
{ }_{1} B_{S y m S U}= & \frac{B^{T}}{2}-\frac{B^{T} \mathbf{s}_{i} \mathbf{s}_{i}^{T}}{2 \mathbf{s}_{i}^{T} \mathbf{s}_{i}}+\frac{B}{2}+\frac{B \mathbf{s}_{i} \mathbf{s}_{i}^{T}}{2 \mathbf{s}_{i}^{T} \mathbf{s}_{i}}-B S S^{+}-\left(S^{+}\right)^{T} S^{T} B^{T}+\frac{\left(S^{+}\right)^{T} S^{T} B^{T} \mathbf{s}_{i} \mathbf{s}_{i}^{T}}{2 \mathbf{s}_{i}^{T} \mathbf{s}_{i}} \\
& +\frac{\left(S^{+}\right)^{T} S^{T} B^{T} S S^{+}}{2}-\frac{\left(S^{+}\right)^{T} S^{T} B \mathbf{s}_{i} \mathbf{s}_{i}^{T}}{2 \mathbf{s}_{i}^{T} \mathbf{s}_{i}}+\frac{\left(S^{+}\right)^{T} S^{T} B S S^{+}}{2}+\frac{\left(S^{+}\right)^{T} S^{T} \mathbf{y}_{i} \mathbf{s}_{i}^{T}}{2 \mathbf{s}_{i}^{T} \mathbf{s}_{i}} \\
& -\frac{\left(S^{+}\right)^{T} S^{T} Y S^{+}}{2}+\left(S^{+}\right)^{T} Y^{T}-\frac{\left(S^{+}\right)^{T} Y^{T} \mathbf{s}_{i} \mathbf{s}_{i}^{T}}{2 \mathbf{s}_{i}^{T} \mathbf{s}_{i}}-\frac{\left(S^{+}\right)^{T} Y^{T} S S^{+}}{2}+\frac{\mathbf{s}_{i} \mathbf{s}_{i}^{T} B^{T}}{2 \mathbf{s}_{i}^{T} \mathbf{s}_{i}} \\
& -\frac{\mathbf{s}_{i} \mathbf{s}_{i}^{T} B^{T} S S^{+}}{2 \mathbf{s}_{i}^{T} \mathbf{s}_{i}}-\frac{\mathbf{s}_{i} \mathbf{s}_{i}^{T} B}{2 \mathbf{s}_{i}^{T} \mathbf{s}_{i}}+\frac{\mathbf{s}_{i} \mathbf{s}_{i}^{T} B S S^{+}}{2 \mathbf{s}_{i}^{T} \mathbf{s}_{i}}-\frac{\mathbf{s}_{i} \mathbf{s}_{i}^{T} Y S^{+}}{2 \mathbf{s}_{i}^{T} \mathbf{s}_{i}}+\frac{\mathbf{s}_{i} \mathbf{y}_{i}^{T} S S^{+}}{2 \mathbf{s}_{i}^{T} \mathbf{s}_{i}}+Y S^{+}
\end{aligned}
$$




$$
\begin{aligned}
{ }_{2} B_{M S \triangleright S y m}= & \frac{B^{T}}{2}-\frac{B^{T} S S^{+}}{2}+\frac{B}{2}-\frac{B S S^{+}}{2}-\frac{\left(S^{+}\right)^{T} S^{T} B^{T}}{2}+\frac{\left(S^{+}\right)^{T} S^{T} B^{T} S S^{+}}{2} \\
& -\frac{\left(S^{+}\right)^{T} S^{T} B}{2}+\frac{\left(S^{+}\right)^{T} S^{T} B S S^{+}}{2}+\left(S^{+}\right)^{T} Y^{T}-\left(S^{+}\right)^{T} Y^{T} S S^{+}+Y S^{+} \\
{ }_{2} B_{S y m S U}= & \frac{B^{T}}{2}-\frac{B^{T} S S^{+}}{2}+\frac{B}{2}-\frac{B S S^{+}}{2}-\frac{\left(S^{+}\right)^{T} S^{T} B^{T}}{2}+\frac{\left(S^{+}\right)^{T} S^{T} B^{T} S S^{+}}{2} \\
& -\frac{\left(S^{+}\right)^{T} S^{T} B}{2}+\frac{\left(S^{+}\right)^{T} S^{T} B S S^{+}}{2}+\frac{\left(S^{+}\right)^{T} S^{T} \mathbf{y}_{i} \mathbf{s}_{i}^{T}}{2 \mathbf{s}_{i}^{T} \mathbf{s}_{i}} \\
& -\frac{\left(S^{+}\right)^{T} S^{T} Y S^{+}}{2}+\left(S^{+}\right)^{T} Y^{T}-\frac{\left(S^{+}\right)^{T} Y^{T} \mathbf{s}_{i} \mathbf{s}_{i}^{T}}{2 \mathbf{s}_{i}^{T} \mathbf{s}_{i}} \\
& -\frac{\left(S^{+}\right)^{T} Y^{T} S S^{+}}{2}-\frac{\mathbf{s}_{i} \mathbf{s}_{i}^{T} Y S^{+}}{2 \mathbf{s}_{i}^{T} \mathbf{s}_{i}}+\frac{\mathbf{s}_{i} \mathbf{y}_{i}^{T} S S^{+}}{2 \mathbf{s}_{i}^{T} \mathbf{s}_{i}}+Y S^{+} \\
{ }_{3} B_{M S \triangleright S y m}= & \frac{B^{T}}{2}-\frac{B^{T} S S^{+}}{2}+\frac{B}{2}-\frac{B S S^{+}}{2}-\frac{\left(S^{+}\right)^{T} S^{T} B^{T}}{2}+\frac{\left(S^{+}\right)^{T} S^{T} B^{T} S S^{+}}{2} \\
& -\frac{\left(S^{+}\right)^{T} S^{T} B}{2}+\frac{\left(S^{+}\right)^{T} S^{T} B S S^{+}}{2}+\left(S^{+}\right)^{T} Y^{T}-\left(S^{+}\right)^{T} Y^{T} S S^{+}+Y S^{+}
\end{aligned}
$$

We find out that (D.1) is equal to (D.3). The sequence converges to a fixed point. The equations (D.1) and (D.2) respectively lead thus to the formulas of Theorems 6 (SUgPSB MS) and 5 (SUgPSB Sym). 